[Article]

\title{
水热法制备 $p-\mathrm{CoFe}_{2} \mathrm{O}_{4} / n-\mathrm{CdS}$ 及其光催化制氢性能
}

\author{
胡海龙 ${ }^{1}$ 王 晟 ${ }^{1, *}$ 侯美顺 ${ }^{1} \quad$ 刘福生 $^{2, *}$ 王田珍 ${ }^{1}$ \\ 李天龙 ${ }^{1}$ 董乾乾 ${ }^{1}$ 张 金金 $^{1}$ \\ ('南京工业大学化工学院, 南京 210009; ${ }^{2}$ 南京林业大学理学院化学与材料科学系, 南京 210037)
}

\begin{abstract}
摘要: 水热法制备了系列 $p-n$ 复合半导体 $p-\mathrm{CoFe}_{2} \mathrm{O}_{4} / n-\mathrm{CdS}$ 。采用 $\mathrm{X}$ 射线衍射 $(\mathrm{XRD})$ 、冷场发射扫描电子显 微镜(SEM)、紫外-可见漫反射光谱(UV-Vis DRS)、透射电镜(TEM)和电化学工作站等对制得的光催化剂进行 了结构和性能表征。研究了 $p-\mathrm{CoFe}_{2} \mathrm{O}_{4} / n-\mathrm{CdS}$ 复合光催化剂的可见光催化制氢性能及光腐蚀性能, 并对光催 化活性的提高、反应条件的影响及光腐蚀行为的抑制机理进行了分析。结果表明: 由于 $\mathrm{CoFe}_{2} \mathrm{O}_{4}$ 和 CdS 两种 窄带隙半导体复合增加了光吸收率; $\mathrm{CdS}$ 独特的树形结构以及 $\mathrm{CoFe}_{2} \mathrm{O}_{4}$ 和 $\mathrm{CdS}$ 二者复合所产生的能带交迭和 内建电场的三重作用, 促进了电子从 $\mathrm{CoFe}_{2} \mathrm{O}_{4}$ 向 $\mathrm{CdS}$ 的迁移, 减少电子-空穴对复合的概率, 增强了光催化 活性。光生电子-空穴对的分离效率以及光催化剂表面吸附性能都对产氢速率有重要影响。 $\mathrm{CH}_{3} \mathrm{OH}$ 水溶液的 $\mathrm{pH}$ 对光催化剂中光生电子-空穴对的分离效率以及光催化剂表面吸附性能都有影响。牺牲剂 $\mathrm{CH}_{3} \mathrm{OH}$ 的加入以 及 $\mathrm{CoFe}_{2} \mathrm{O}_{4}$ 和 $\mathrm{CdS}$ 二者复合所产生的能带交迭和内建电场的作用都对 $\mathrm{CdS}$ 的光腐蚀起了抑制作用, 后者的 抑制效果更好。
\end{abstract}

关键词: $\mathrm{CoFe}_{2} \mathrm{O}_{4} ; \mathrm{CdS}$; 光催化; 制氢; 光腐蚀

中图分类号: 0643

\section{Preparation of $p-\mathrm{CoFe}_{2} \mathrm{O}_{4} / n-\mathrm{CdS}$ by Hydrothermal Method and Its Photocatalytic Hydrogen Production Activity}

\author{
HU Hai-Long1 $\quad$ WANG Sheng ${ }^{1, *} \quad$ HOU Mei-Shun ${ }^{1} \quad$ LIU Fu-Sheng ${ }^{2, *} \quad$ WANG Tian-Zhen ${ }^{1}$ \\ LI Tian-Long1 $\quad$ DONG Qian-Qian ${ }^{1} \quad$ ZHANG Xin ${ }^{1}$ \\ ('College of Chemical Engineering, Nanjing Tech University, Nanjing 210009, P. R. China; \\ ${ }^{2}$ Department of Chemistry and Materials Science, College of Science, Nanjing Forestry University, Nanjing 210037, P. R. China)
}

\begin{abstract}
A series of $p-n$ coupled $p-\mathrm{CoFe}_{2} \mathrm{O}_{4} / n$-CdS photocatalysts were prepared by a hydrothermal method. The structure and properties of $p-\mathrm{CoFe}_{2} \mathrm{O}_{4} / n$-CdS were characterized by X-ray diffraction (XRD), scanning electron microscopy (SEM), ultraviolet-visible diffuse reflectance spectroscopy (UV-Vis DRS), transmission electron microscopy (TEM) and the electrochemical workstation. The photocatalytic activities of $p-\mathrm{CoFe}_{2} \mathrm{O}_{4} / n-$ CdS were evaluated by photocatalytic hydrogen production under visible light irradiation. The effects of photocorrosion of $\mathrm{CdS}$ in $p-\mathrm{CoFe}_{2} \mathrm{O}_{4} / n$-CdS were investigated by analyzing the cadmium concentration of the supernatant collected after the photocatalytic reactions. The mechanism of the catalytic activity enhancement, effects of reaction conditions on the photocatalytic hydrogen evolution rate, and cadmium leakage resistance are discussed. The results show that $p-\mathrm{CoFe}_{2} \mathrm{O}_{4} / n-\mathrm{CdS}$ exhibits higher photocatalytic activity attributed to
\end{abstract}

Received: August 1, 2016; Revised: November 23, 2016; Published online: November 24, 2016.

"Corresponding authors. WANG Sheng, Email: w_angsheng@njtech.edu.cn,w_angshengnj@163.com; Tel: +86-25-83587197.

LIU Fu-Sheng, Email: 1fs039270@163.com; Tel: +86-25-85427625.

The project was supported by the National Natural Science Foundation of China (50876047), Top-notch Academic Programs Project of Jiangsu Higher Education Institutions, China (TAPP, PPZY2015A044) and Scientific Research Innovation Program of Graduate in Colleges and Universities of Jiangsu Province, China (SJLX15-0346).

国家自然科学基金(50876047), 江苏高校品牌专业建设工程项目(TAPP, PPZY2015A044)和江苏省普通高校研究生科研创新计划项目(SJLX150346)资助

(C) Editorial office of Acta Physico-Chimica Sinica 
stronger light absorption by the two types of narrow band gap semiconductor $\mathrm{CoFe}_{2} \mathrm{O}_{4}$ and $\mathrm{CdS}$, the formation of a "forest-like" structure of $\mathrm{CdS}$ and rapid electron transfer from $\mathrm{CoFe}_{2} \mathrm{O}_{4}$ to $\mathrm{CdS}$, resulting from band overlap and an inner electric field in $p-\mathrm{CoFe}_{2} \mathrm{O}_{4} / n$-CdS, to reduce the probability of electron/hole pair recombination. Both the separation efficiency of photo-generated electron-hole pairs and the adsorption performance of photocatalysts had an important influence on the hydrogen production rate. The $\mathrm{pH}$ of the $\mathrm{CH}_{3} \mathrm{OH}$ aqueous solution influenced the separation efficiency of photogenerated electron-hole pairs and the adsorption properties of the photocatalyst. $p-\mathrm{CoFe}_{2} \mathrm{O}_{4} / n$-CdS also exhibited resistance against cadmium leakage under light irradiation owing to the presence of methanol in the reaction solution, the band overlap of the semiconductors and the inner electric field in $p-\mathrm{CoFe}_{2} \mathrm{O}_{4} / n-\mathrm{CdS}$. The band overlap and inner electric field had the most influence on the cadmium leakage resistance.

Key Words: $\mathrm{CoFe}_{2} \mathrm{O}_{4} ;$ CdS; Photocatalysis; Hydrogen production; Photocorrosion

\section{1 引言}

21 世纪以来, 随着能源危机的日益严重, 半 导体材料可见光催化制氢逐渐引起了人们的关 注。在国内、外已研究的各类半导体中, 对可见 光敏感的半导体 $\mathrm{CdS}$ 最受关注。但由于 $\mathrm{CdS}$ 光催化 分解水制氢的效率不高 ${ }^{1}$, 且产生的空穴易与 $\mathrm{CdS}$ 反应, 即发生光腐蚀, 其腐蚀产物 $\mathrm{Cd}^{2+}$ 不仅造成二 次环境污染, 而且会降低 CdS 的使用寿命和光催 化效率 ${ }^{2,3}$, 因此, 提高 $\mathrm{CdS}$ 的可见光催化效率、降 低甚至消除 CdS 的光腐蚀成为热点研究课题。目 前常用的抑制 CdS 光生电子-空穴对复合, 促进光 生电荷有效分离的途径有离子掺杂 4,5 、贵金属负 载 6,7 、非金属负载 ${ }^{8}$ 、半导体复合 ${ }^{9-11}$ 和制备固溶 体 ${ }^{12,13}$ 等。磁性 $\mathrm{CoFe}_{2} \mathrm{O}_{4}$ 也是一种性能优良的窄带隙 半导体材料, 对可见光敏感, 表现出了良好的可 见光催化活性而引起了人们的关注。但是 $\mathrm{CoFe}_{2} \mathrm{O}_{4}$ 的禁带宽度窄, 生成的电子空穴对极易复合。目 前, 主要是通过与其它半导体复合来改善 $\mathrm{CoFe}_{2} \mathrm{O}_{4}$ 的光催化效率, 已有的报道表明, $\mathrm{CoFe}_{2} \mathrm{O}_{4}$ 和 $\mathrm{CdS}$ 复合, 可有效提高光催化降解甲基蓝和四环素抗 生素的效率 ${ }^{14-17}$ 。虽然 $\mathrm{CoFe}_{2} \mathrm{O}_{4} / \mathrm{CdS}$ 光催化降解有 机物已有文献报道, 但是将 $\mathrm{CoFe}_{2} \mathrm{O}_{4} / \mathrm{CdS}$ 应用于可 见光催化制氢过程, 探讨提高产氢性能和抑制光 腐蚀的机制方面的研究还不够深入。

按导电载流子的不同, 光催化半导体材料也 可分为 $p$ 型半导体和 $n$ 型半导体两类。由半导体物 理学理论可知, $n$ 型半导体和 $p$ 型半导体复合时, 接触界面两侧会分别形成带正电薄层和带负电薄 层, 两薄层间便产生了一个内建电场, 即形成了 带正电薄层和带负电薄层的 $p-n$ 结。研究结果表明 $p-n$ 复合半导体可促进光生电子-空穴对迅速分离, 有效减少电子-空穴对复合的概率, 从而提高光催
化活性 ${ }^{18,19}$ 。

$\mathrm{CoFe}_{2} \mathrm{O}_{4}$ 和 $\mathrm{CdS}$ 分别为 $p$ 型半导体和 $n$ 型半导 体, 本文用两种窄带隙半导体 $p-\mathrm{CoFe}_{2} \mathrm{O}_{4}$ 与 $n-\mathrm{CdS}$ 复合制备 $p-n$ 复合半导体光催化剂 $p-\mathrm{CoFe}_{2} \mathrm{O}_{4} / n$ $\mathrm{CdS}$, 考察 $\mathrm{CH}_{3} \mathrm{OH}$ 水溶液中 $p-\mathrm{CoFe}_{2} \mathrm{O}_{4} / n-\mathrm{CdS}$ 可见 光催化制氢的性能和循环使用性能, 并对其光催 化活性的提高以及光腐蚀性能的抑制进行了机制 分析。

\section{2 实验部分}

\section{1 主要试剂}

氯化镉 $\left(\mathrm{CdCl}_{2} \cdot x \mathrm{H}_{2} \mathrm{O}\right)$ 、氯化钴 $\left(\mathrm{CoCl}_{2} \cdot 6 \mathrm{H}_{2} \mathrm{O}\right)$ 、 氯化铁 $\left(\mathrm{FeCl}_{3} \cdot 6 \mathrm{H}_{2} \mathrm{O}\right)$ 、氢氧化钠 $(\mathrm{NaOH})$ 和硫艮 $\left(\mathrm{CN}_{2} \mathrm{H}_{4} \mathrm{~S}\right)$ 等试剂均为分析纯, 购于国药集团化学试 剂有限公司。所用溶液用去离子水配制。

\section{$2.2 p-\mathrm{CoFe}_{2} \mathrm{O}_{4} / n-\mathrm{CdS}$ 光催化剂的制备}

将 $\mathrm{CoCl}_{2} \cdot 6 \mathrm{H}_{2} \mathrm{O}$ 和 $\mathrm{FeCl}_{3} \cdot 6 \mathrm{H}_{2} \mathrm{O}$ 分别溶解于去离 子水中, 再将已配好的 $\mathrm{NaOH}$ 溶液滴加至其中 $\left(\mathrm{CoCl}_{2} \cdot 6 \mathrm{H}_{2} \mathrm{O} 、 \mathrm{FeCl}_{3} \cdot 6 \mathrm{H}_{2} \mathrm{O}\right.$ 和 $\mathrm{NaOH}$ 的摩尔比为 1 : $2: 10)$, 摚拌 $0.5 \mathrm{~h}$, 得到 $\mathrm{CoFe}_{2} \mathrm{O}_{4}$ 的前驱体溶液, 记为溶液 $\mathrm{A}$ 。将硫腿溶解于去离子水中, 然后滴加 到已经配好的 $\mathrm{CdCl}_{2} \cdot x \mathrm{H}_{2} \mathrm{O}$ 溶液中 (硫脲和 $\mathrm{CdCl}_{2}$. $x \mathrm{H}_{2} \mathrm{O}$ 的摩尔比为 $\left.1: 1\right)$, 搅拌 $0.5 \mathrm{~h}$, 得到 $\mathrm{CdS}$ 的前 驱体溶液, 记为溶液 $\mathrm{B}$ 。将溶液 $\mathrm{A}$ 加入到溶液 $\mathrm{B}$ 中, 混合液转移到 $100 \mathrm{~mL}$ 的聚四氟乙烯反应釜 中, 在 $180^{\circ} \mathrm{C}$ 条件下反应 $20 \mathrm{~h}$, 产物经过水洗、研 磨得到 $p-\mathrm{CoFe}_{2} \mathrm{O}_{4} / n-\mathrm{CdS}$ 粉末。为了比较, 在同样 条件下分别制备了 $\mathrm{CdS}$ 和 $\mathrm{CoFe}_{2} \mathrm{O}_{4}$ 。

\section{3 光催化剂的性能表征}

$\mathrm{X}$ 射线衍射(XRD)表征在 D8 Advance 型(德国 Bruker 公司) $X$ 射线粉末衍射仪上进行, 入射光为 $\mathrm{Cu}$ 靶 $K_{\alpha}$ 线, 加速电压 $40 \mathrm{kV}$, 电流 $30 \mathrm{~mA}$, 扫描范 
围为 $20^{\circ}-80^{\circ}$ 。扫描电子显微镜 $(\mathrm{SEM})$ 表征和能量 色散 X 射线谱(EDS)分析均在 S-4800 型冷场发射扫 描电子显微镜(日本 Hitachi 公司)上进行。透射电子 显微镜(TEM)表征在 JEM-2010 UHR 型透射电镜(日 本电子公司)上进行。紫外-可见漫反射光谱(UV-Vis DRS)采用Lambda 950型分光光度计(美国Perkin Elmer 公司)测量, 以 $\mathrm{BaSO}_{4}$ 为参比, 扫描范围 300-800 $\mathrm{nm}$ 。用 $\mathrm{pH}$ 值漂移法测量催化剂的零电位 $\mathrm{pH}$ 值 $\left(\mathrm{pH}_{\mathrm{pzc}}\right)$ 。用 $0.1 \mathrm{~mol} \cdot \mathrm{L}^{-1}$ 的盐酸和 $0.1 \mathrm{~mol} \cdot \mathrm{L}^{-1}$ 氢氧化 钠调节 $\mathrm{pH}$, 具体测试方法同文献 ${ }^{13}$ 。以每种溶液 未加催化剂时的初始 $\mathrm{pH}$ 值 $\left(\mathrm{pH}_{\mathrm{i}}\right)$ 为横坐标, 加入光 催化剂后振荡 $24 \mathrm{~h}$ 后的最终 $\mathrm{pH}$ 值 $\left(\mathrm{pH}_{\mathrm{f}}\right)$ 为纵坐标作 图, 所得曲线与直线 $\mathrm{pH}_{\mathrm{i}}=\mathrm{pH}_{\mathrm{f}}$ 交点的纵坐标值, 即为所测催化剂的零电位 $\mathrm{pH}$ 值。电化学性能测试 体系为三电极体系, 工作电极基体材料是氧化铟 锡(ITO) 导电玻璃(面积为 $10 \mathrm{~mm} \times 10 \mathrm{~mm}$ ), 光催化 剂粉末通过物理沉积法沉积在 ITO 导电玻璃上 ${ }^{21}$ 。 $\mathrm{Pt}$ 片电极为辅助电极, 饱和甘录电极 (SCE) 为参比 电极, 电化学性能测试的电解液均为 $0.1 \mathrm{~mol} \cdot \mathrm{L}^{-1}$ 的 $\mathrm{Na}_{2} \mathrm{SO}_{4}$ 。测试均在室温下进行, 外照光源为 350 $\mathrm{W}$ 氙灯(南京歼鹰展翅科技有限公司)。CHI660E 型 电化学工作站(上海辰华仪器有限公司), 对 MottSchottky 及瞬态光电流响应曲线图进行数据采集, 其中瞬态光电流响应曲线的光照时间间隔为 200 $\mathrm{s}$, 所加偏压为 0, Mott-Schottky 图的测试电位 $(E)$ 范围为 $-0.7-0.6 \mathrm{~V}$, 振幅为 $5 \mathrm{mV}$, 频率为 500 $\mathrm{Hz}$ 。

\section{4 光催化活性评价}

制备的光催化剂的光催化活性是通过可见光 催化产氢速率进行评价。光催化反应在 $100 \mathrm{~mL}$ 侧 面接受光照的石英烧瓶中进行, 光源为 $350 \mathrm{~W}$ 氙 灯, 光照强度为 $4.0 \mathrm{~mW} \cdot \mathrm{cm}^{-2}$, 滤光片去掉波长 $400 \mathrm{~nm}$ 以下的紫外光。将 $0.1 \mathrm{~g} \cdot \mathrm{L}^{-1}$ 光催化剂加入 到 $\mathrm{CH}_{3} \mathrm{OH}$ 水溶液 $\left(\mathrm{CH}_{3} \mathrm{OH}\right.$ 体积分数为 $10 \%(\varphi$, 体 积分数)) 中, 反应温度为 $35{ }^{\circ} \mathrm{C}$ 。光照前, 悬浮液 先超声 $15 \mathrm{~min}$, 然后向其中充入高纯 $\mathrm{N}_{2} 30 \mathrm{~min}$, 以确保反应是在无溶解氧的条件下进行。制备得 到的 $\mathrm{H}_{2}$ 用 FULI 9790 型气相色谱仪(温岭市福立分 析仪器有限公司)进行检测。检测条件为: 填充柱 中固定相为 Porapak $\mathrm{Q}$, 高纯 $\mathrm{N}_{2}$ 作载气, $\mathrm{N}_{2}$ 流速为 $35 \mathrm{~mL} \cdot \mathrm{min}^{-1}$, 热导检测器, 柱温 $50^{\circ} \mathrm{C}$, 汽化温度 $50^{\circ} \mathrm{C}$, 检测温度 $50^{\circ} \mathrm{C}$ 。每次实验开始前, 均进行 暗反应实验和空白实验, 结果显示暗反应和空白
实验均不产生 $\mathrm{H}_{2}$ 。

\section{3 结果与讨论}

\section{$3.1 p-\mathrm{CoFe}_{2} \mathrm{O}_{4} / n-\mathrm{CdS}$ 光催化剂的表征}

\section{1 .1 光催化剂的 XRD 分析}

图 1 是 $\mathrm{CdS} 、 \mathrm{CoFe}_{2} \mathrm{O}_{4}$ 和不同配比 $p-\mathrm{CoFe}_{2} \mathrm{O}_{4} / n-$ $\mathrm{CdS}$ 样品的 $\mathrm{X}$ 射线粉末衍射图。由图可以清晰的看 出, $\mathrm{CdS}$ 与 $\mathrm{CoFe}_{2} \mathrm{O}_{4}$ 复合后, $\mathrm{CdS}$ 的晶型没有发生 改变。在衍射角 $2 \theta$ 依次为 $24.81^{\circ}, 26.51^{\circ}$, $28.18^{\circ}, 36.62^{\circ}, 43.68^{\circ}, 47.84^{\circ}$ 和 $51.82^{\circ}$ 处对应着 六方纤锌矿 $\mathrm{CdS}$ 的 (100), (002), (101), (102), (110), (103) 和 (112) 晶面 (JCPDS 41-1049)。当 $\mathrm{CoFe}_{2} \mathrm{O}_{4}$ 含量低于 $6.0 \%$ ( $w$, 质量分数)时, 在复合 光催化剂 $\mathrm{CoFe}_{2} \mathrm{O}_{4} / \mathrm{CdS}$ 的 XRD 衍射谱图中, 没有 观察到 $\mathrm{CoFe}_{2} \mathrm{O}_{4}$ 的特征衍射峰, 这可能是由于 $\mathrm{CoFe}_{2} \mathrm{O}_{4}$ 含量较低, 并均匀分散在催化剂表面, 不 在仪器检测的阈值范围内。当 $\mathrm{CoFe}_{2} \mathrm{O}_{4}$ 含量大于等 于 $6.0 \%(w)$ 时, $\mathrm{XRD}$ 衍射谱图中可观察到 $\mathrm{CoFe}_{2} \mathrm{O}_{4}$ 的(311)晶面(JCPDS 22-1068)的特征衍射峰, 且随 着 $\mathrm{CoFe}_{2} \mathrm{O}_{4}$ 含量的增加, (311) 晶面的特征衍射峰强 度增加, 没有其他杂质峰。

\section{1 .2 光催化剂的形貌分析}

$\mathrm{CdS}$ 和 $6.0 \%(w) \mathrm{CoFe}_{2} \mathrm{O}_{4} / \mathrm{CdS}$ 的 SEM 照片见图 2, 由图 2(A)可以看出, $\mathrm{CdS}$ 的形貌为树形状, 边 缘清晰, 长度在 $2-4 \mu \mathrm{m}$ 。由图 2(B) 可以看出: $6.0 \%(w) \mathrm{CoFe}_{2} \mathrm{O}_{4} / \mathrm{CdS}$ 样品的 SEM 图中明显可见有 100-200 nm 左右的球状小颗粒附着在树形状 CdS

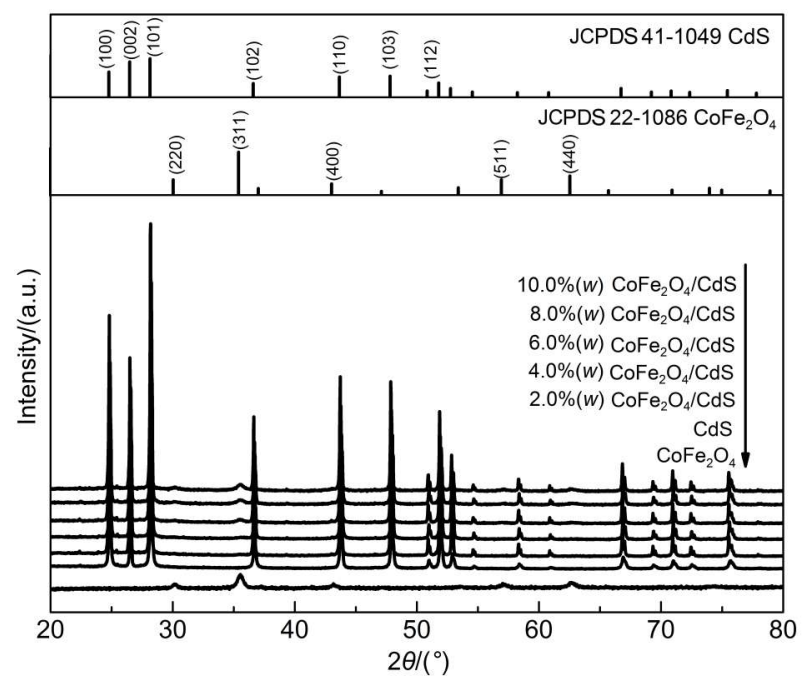

图 $1 \mathrm{CdS} 、 \mathrm{CoFe}_{2} \mathrm{O}_{4}$ 和 $p-\mathrm{CoFe}_{2} \mathrm{O}_{4} / n-\mathrm{CdS}$ 的 $\mathrm{XRD}$ 图 Fig.1 X-ray diffraction (XRD) patterns of $\mathrm{CdS}, \mathrm{CoFe}_{2} \mathrm{O}_{4}$ and $p-\mathrm{CoFe}_{2} \mathrm{O}_{4} / n-\mathrm{CdS}$ 


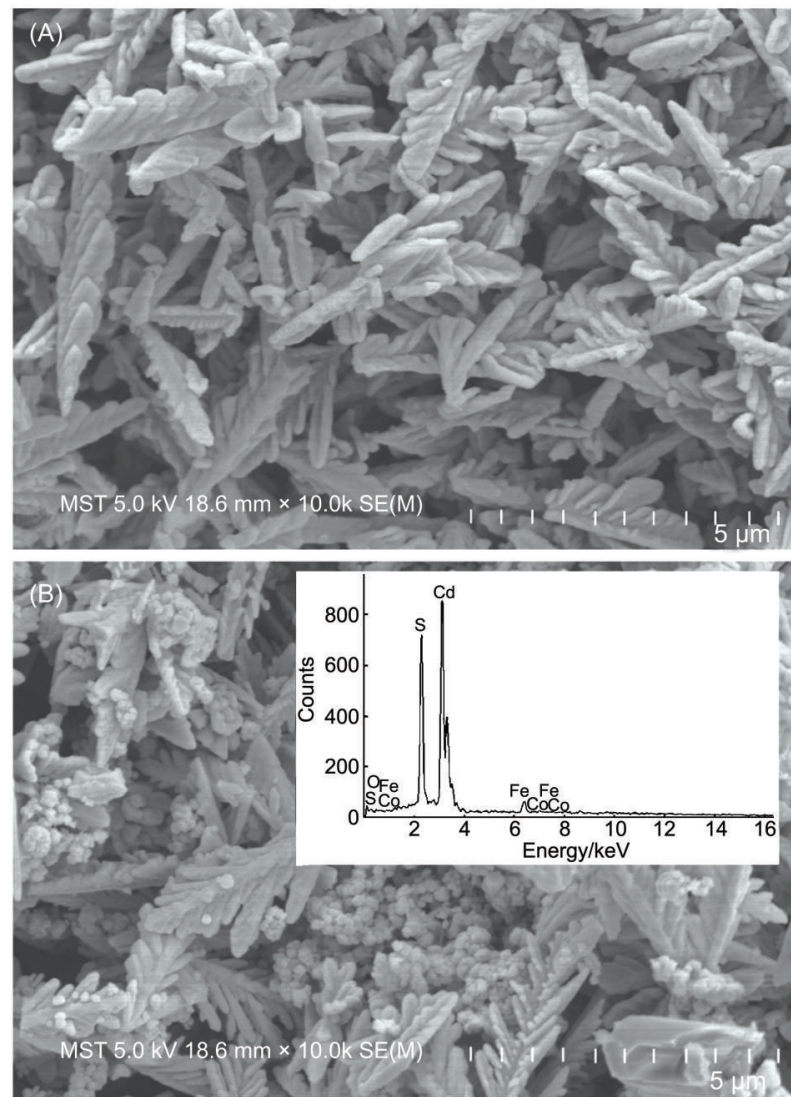

图 2 (A) $\mathrm{CdS}$ 和(B) $6.0 \%$ (w) $\mathrm{CoFe}_{2} \mathrm{O}_{4} / \mathrm{CdS}$ 的 SEM 照片 Fig.2 Scanning electron microscope (SEM) images of (A) $\mathrm{CdS}$ and (B) $6.0 \%$ (w) $\mathrm{CoFe}_{2} \mathrm{O}_{4} / \mathrm{CdS}$ Inset in (B) shows the corresponding energy dispersive $\mathrm{X}$-ray spectroscopy (EDS).

的表面。与 $\mathrm{CdS}$ 进行比较, 可以推断这些球状颗 粒为 $\mathrm{CoFe}_{2} \mathrm{O}_{4}$ 。从 $\mathrm{EDS}$ 分析结果可以看出: 产物由 $\mathrm{Co} 、 \mathrm{Fe} 、 \mathrm{O} 、 \mathrm{Cd}$ 和 $\mathrm{S}$ 五种元素组成，没有其他杂 质, 与 XRD 的分析结果一致, 进一步证实了产物 是 $p-\mathrm{CoFe}_{2} \mathrm{O}_{4} / n-\mathrm{CdS}$ 。

为了确定 $p-\mathrm{CoFe}_{2} \mathrm{O}_{4} / n-\mathrm{CdS}$ 异质结的形貌和结 构, 对制备样品 $6.0 \%(w) \mathrm{CoFe}_{2} \mathrm{O}_{4} / \mathrm{CdS}$ 进行了 $\mathrm{TEM}$ 分析和 HRTEM 分析。图 3(A)为 6.0\% (w) $\mathrm{CoFe}_{2} \mathrm{O}_{4} /$ $\mathrm{CdS}$ 异质结的 TEM 图像, 图中显示的形貌为树形 状，与上述 SEM 分析结果一致。图 3(B) 为 $6.0 \%$ (w) $\mathrm{CoFe}_{2} \mathrm{O}_{4} / \mathrm{CdS}$ 的 HRTEM 图, 图中可见两种不同 的晶相, 其中晶格间距为 $0.2947 \mathrm{~nm}$ 对应的是 $\mathrm{CoFe}_{2} \mathrm{O}_{4}$ 的(112)晶面, 而晶格间距为 $0.18998 \mathrm{~nm}$ 对 应的是 $\mathrm{CdS}$ 的(103)晶面。 $\mathrm{CoFe}_{2} \mathrm{O}_{4}$ 和 $\mathrm{CdS}$ 间的紧密 结合可以确定, 在实验过程中确有 $p-\mathrm{CoFe}_{2} \mathrm{O}_{4} / n$ $\mathrm{CdS}$ 异质结产生, 这将有利于电子和空穴借助于异 质结的进行电荷传输, 从而有利于电子-空穴对的 有效分离。
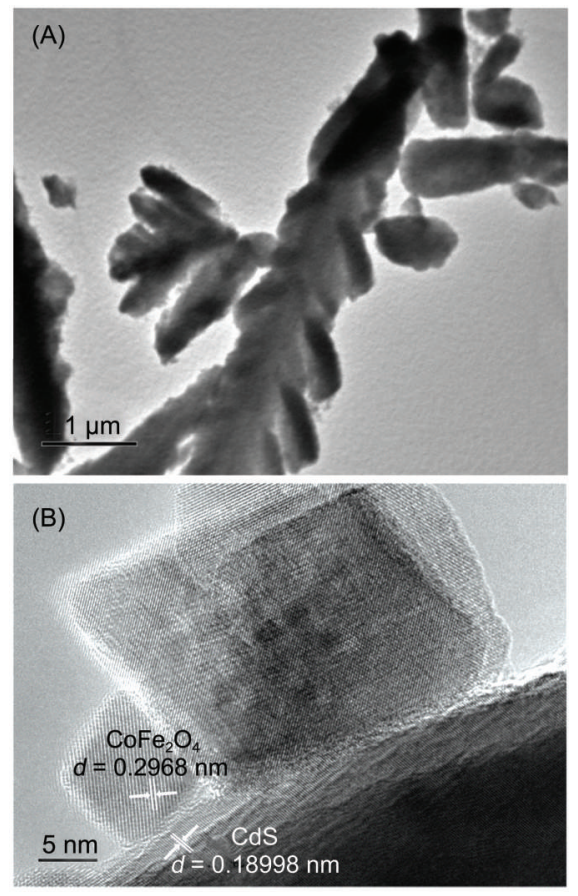

图 $36.0 \%(w) \mathrm{CoFe}_{2} \mathrm{O}_{4} / \mathrm{CdS}$ 的(A) TEM 和 (B) HRTEM照片

Fig.3 (A) TEM image and (B) high resolution TEM (HRTEM) image of $6.0 \%(w) \mathrm{CoFe}_{2} \mathrm{O}_{4} / \mathrm{CdS}$

\subsection{3 光催化剂的 UV-Vis DRS 分析}

$\mathrm{CdS} 、 \mathrm{CoFe}_{2} \mathrm{O}_{4}$ 以及不同配比 $p-\mathrm{CoFe}_{2} \mathrm{O}_{4} / n-\mathrm{CdS}$ 的 UV-Vis DRS 图谱见图 4(A)。从图 4(A)上可以看 出: $\mathrm{CoFe}_{2} \mathrm{O}_{4}$ 对波长低于 $800 \mathrm{~nm}$ 的紫外光和可见光 均有吸收, 而 $\mathrm{CdS}$ 只能吸收波长小于 $600 \mathrm{~nm}$ 的紫 外光和可见光, $p-\mathrm{CoFe}_{2} \mathrm{O}_{4} / n-\mathrm{CdS}$ 则对波长低于 $800 \mathrm{~nm}$ 的紫外光和可见光均有吸收, 且随着 $\mathrm{CoFe}_{2} \mathrm{O}_{4}$ 含量的增加, 在 600-800 nm 范围内的可 见光区的吸收强度逐渐增强, 吸收带边明显蓝 移, 这种连续的蓝移现象是由于 $\mathrm{CoFe}_{2} \mathrm{O}_{4}$ 颗粒的存 在引起的。

半导体材料禁带宽度 $\left(E_{\mathrm{g}}\right)$ 可以利用 $(\alpha h v)^{n} \propto$ $\left(h v-E_{\mathrm{g}}\right)$ 的关系曲线求得 $(\alpha$ 为吸收系数, $h$ 为普朗 克常数, $v$ 为入射光频率, $n$ 为参数, 对于直接跃 迁半导体 $\mathrm{CdS}$ 和 $\mathrm{CoFe}_{2} \mathrm{O}_{4}, n$ 取 $2^{23,24}$ )。以 $(\alpha h v)^{2}$ 对 $h v$ 作图, 见图 4(B), 曲线中直线部分的切线在 $h v$ 轴 上的交点即为 $E_{\mathrm{g}}$ 值, 计算得到 $\mathrm{CdS}$ 和 $\mathrm{CoFe}_{2} \mathrm{O}_{4}$ 的禁 带宽度分别为 2.25 和 $1.19 \mathrm{eV}$ 。

\section{1 .4 光催化剂零电位 $\mathrm{pH}$ 值的分析}

图 5 为用 $\mathrm{pH}$ 值漂移法得到的 $\mathrm{CdS}$ 和 6.0\% (w) $\mathrm{CoFe}_{2} \mathrm{O}_{4} / \mathrm{CdS}$ 的 $\mathrm{pH}_{\mathrm{f}}$ 随 $\mathrm{pH}_{\mathrm{i}}$ 变化曲线图, 其中虚线为 直线 $\mathrm{pH}_{\mathrm{i}}=\mathrm{pH}_{\mathrm{f}}$ 。从图 5 可以看出, $\mathrm{CdS}$ 和 $6.0 \%(w)$ 

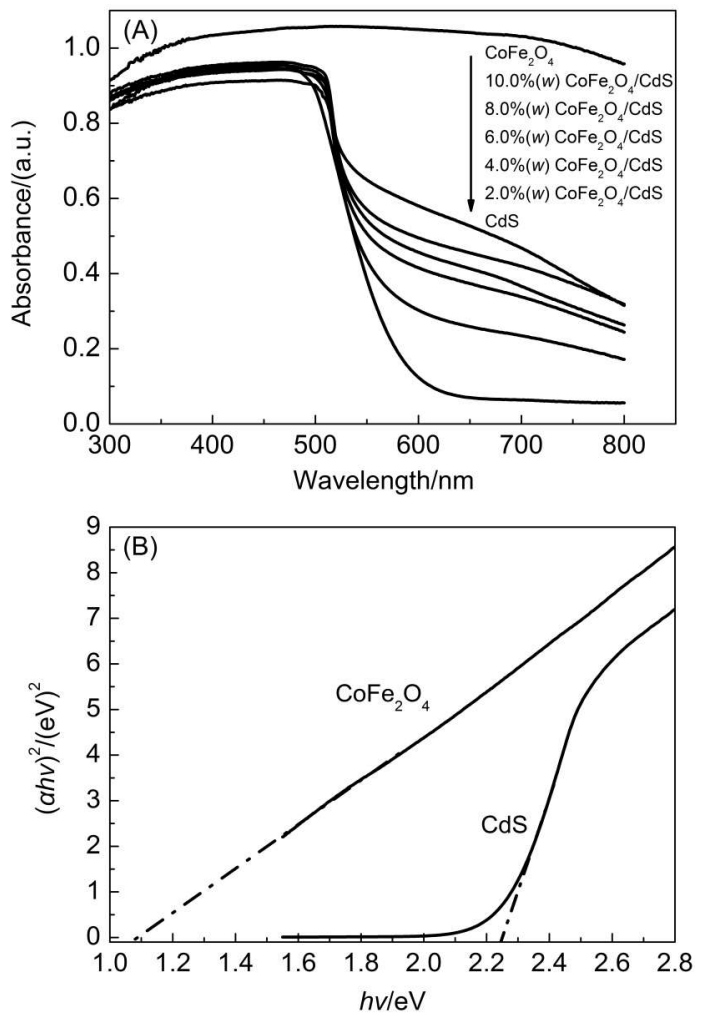

图 4 (A) $\mathrm{CdS} 、 \mathrm{CoFe}_{2} \mathrm{O}_{4}$ 和 $p-\mathrm{CoFe}_{2} \mathrm{O}_{4} / n-\mathrm{CdS}$ 的紫外-可见 漫反射光谱; (B) $\mathrm{CdS}$ 和 $\mathrm{CoFe}_{2} \mathrm{O}_{4}$ 的 $(\alpha h v)^{2}$ 与 $h v$ 关系曲线

Fig.4 (A) Ultraviolet-visible diffuse reflectance spectra (UV-Vis DRS) of CdS, $\mathrm{CoFe}_{2} \mathrm{O}_{4}$ and $\boldsymbol{p}-\mathrm{CoFe}_{2} \mathrm{O}_{4} / n-\mathrm{CdS}$;

(B) $(\alpha h v)^{2}$ versus hv plots of $\mathrm{CdS}$ and $\mathrm{CoFe}_{2} \mathrm{O}_{4}$

$\mathrm{CoFe}_{2} \mathrm{O}_{4} / \mathrm{CdS}$ 的零电位 $\mathrm{pH}$ 值分别为 6.4 和 6.1 。当溶 液的初始 $\mathrm{pH}_{\mathrm{i}}$ 值低于 $\mathrm{CdS}$ 的 $\mathrm{pH}_{\mathrm{pzc}}$ 时, $\mathrm{CdS}$ 的 $\mathrm{pH}_{\mathrm{f}}$ 稍 高于 $\mathrm{pH}_{\mathrm{i}}$, 即加入 $\mathrm{CdS}$ 使溶液的酸度有所降低, 说 明在 $\mathrm{CdS}$ 表面存在着碱性位; 而当溶液初始 $\mathrm{pH}_{\mathrm{i}}$ 值 高于 $\mathrm{CdS}$ 的 $\mathrm{pH}_{\mathrm{pzc}}$ 时, $\mathrm{pH}_{\mathrm{f}}$ 要稍低于 $\mathrm{pH}_{\mathrm{i}}$, 即加入 $\mathrm{CdS}$ 使溶液的碱性有所降低, 说明在 $\mathrm{CdS}$ 表面存在着

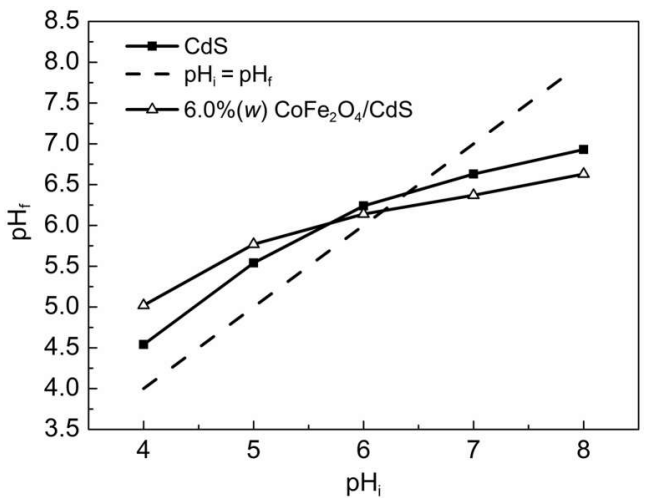

图 $5 \mathrm{pH}$ 漂移法测得的 $\mathrm{CdS}$ 和 $6.0 \%(w) \mathrm{CoFe}_{2} \mathrm{O}_{4} / \mathrm{CdS}$ 的 零电位 $\mathrm{pH}$ 值

Fig.5 $\mathrm{pH}_{\mathrm{pzc}}$ obtained by $\mathrm{pH}$ drift method for $\mathrm{CdS}$ and $6.0 \%$ (w) $\mathrm{CoFe}_{2} \mathrm{O}_{4} / \mathrm{CdS}$
酸性位。由图 5 还可以看出, $6.0 \%(w) \mathrm{CoFe}_{2} \mathrm{O}_{4} /$ $\mathrm{CdS}$ 的 $\mathrm{pH}_{\mathrm{f}}$ 随 $\mathrm{pH}_{\mathrm{i}}$ 的变化趋势与 $\mathrm{CdS}$ 的变化相似, 但 当 $\mathrm{pH}_{\mathrm{i}}$ 低于 $6.0 \%(w) \mathrm{CoFe}_{2} \mathrm{O}_{4} / \mathrm{CdS}$ 的 $\mathrm{pH}_{\mathrm{pzc}}$ 时, 其 $\mathrm{pH}_{\mathrm{f}}$ 比 $\mathrm{pH}_{\mathrm{i}}$ 更高, 而 $\mathrm{pH}_{\mathrm{i}}$ 高于 $6.0 \%(w) \mathrm{CoFe}_{2} \mathrm{O}_{4} / \mathrm{CdS}$ 的 $\mathrm{pH}_{\mathrm{pzc}}$ 时, 其 $\mathrm{pH}_{\mathrm{f}}$ 比 $\mathrm{pH}_{\mathrm{i}}$ 更低, 说明复合 $\mathrm{CoFe}_{2} \mathrm{O}_{4}$ 后光 催化剂表面的酸性位和碱性位的浓度均增大。

\section{1 .5 光催化剂的电化学性能}

$\mathrm{CdS}$ 和 $\mathrm{CoFe}_{2} \mathrm{O}_{4}$ 的 Mott-Schottky 曲线如图 6 所 示。由 Mott-Schottky 曲线可以看出: CdS 的 MottSchottky 曲线的斜率是正, $\mathrm{CoFe}_{2} \mathrm{O}_{4}$ 的 Mott-Schottky 曲线的斜率是负, 这表明 $\mathrm{CdS}$ 是 $n$ 型半导体, $\mathrm{CoFe}_{2} \mathrm{O}_{4}$ 是 $p$ 型半导体。 $\mathrm{CdS}$ 和 $\mathrm{CoFe}_{2} \mathrm{O}_{4}$ 的 MottSchottky 曲线的切线在横坐标上的截距分别 是 -0.48 和 $0.38 \mathrm{eV}$, 说明 $\mathrm{CdS}$ 和 $\mathrm{CoFe}_{2} \mathrm{O}_{4}$ 的平带电 位分别是 -0.48 和 $0.38 \mathrm{eV}$ 。对于 $n$ 型半导体, 导带 电位比平带电位负约 $0.1 \mathrm{eV}^{25}$, 因此, $\mathrm{CdS}$ 的导带 电位是 $-0.58 \mathrm{eV}$, 由图 4(B) 知其禁带宽度是 2.25 $\mathrm{eV}$, 故 $\mathrm{CdS}$ 的价带电位是 $1.67 \mathrm{eV}$; 而对于 $p$ 型半 导体, 其价带电位比其平带电位正约 $0.1 \mathrm{eV}^{26}$, 因 此, $\mathrm{CoFe}_{2} \mathrm{O}_{4}$ 的价带电位是 $0.48 \mathrm{eV}$, 由图 4(b) 知其 禁带宽度是 $1.19 \mathrm{eV}$, 进而算出 $\mathrm{CoFe}_{2} \mathrm{O}_{4}$ 的导带电位 是 $-0.71 \mathrm{eV}$ 。 $\mathrm{CdS}$ 和 $\mathrm{CoFe}_{2} \mathrm{O}_{4}$ 的导带电位均比 $\mathrm{H}^{+} / \mathrm{H}_{2}$ 的还原电位负, 两者都具有光催化分解水制氢的 能力。

图 7 所示为样品的瞬态光电流响应曲线。光电 流的大小可反映电荷迁移的快慢程度, 光电流越 大, 则电荷迁移越快 ${ }^{27}$ 。由图可知, 随着 $\mathrm{CoFe}_{2} \mathrm{O}_{4}$ 含量的增加, 光电流的大小呈现出现增加后减小 的趋势, $\mathrm{CoFe}_{2} \mathrm{O}_{4}$ 的含量为 $6.0 \%(w)$ 时, 光电流的 值最大。

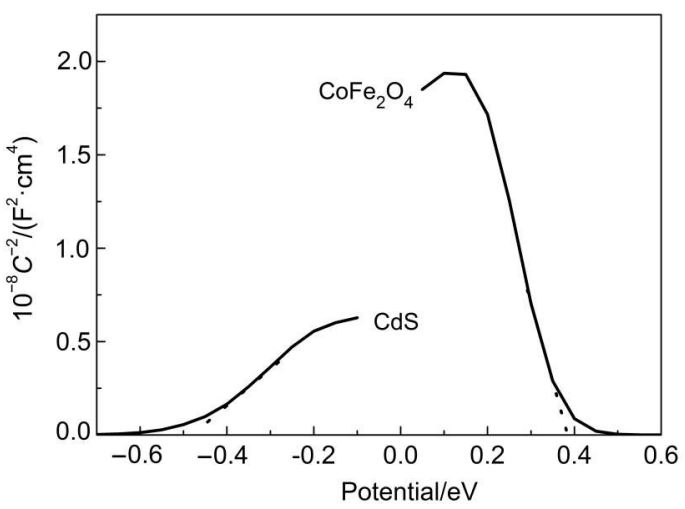

图 $6 \mathrm{CoFe}_{2} \mathrm{O}_{4}$ 和 $\mathrm{CdS}$ 在 $0.1 \mathrm{~mol} \cdot \mathrm{L}^{-1} \mathrm{Na}_{2} \mathrm{SO}_{4}$ 溶液中的 Mott-Schottky 曲线

Fig.6 Mott-Schottky curves for $\mathrm{CoFe}_{2} \mathrm{O}_{4}$ and $\mathrm{CdS}$ in $0.1 \mathrm{~mol} \cdot \mathrm{L}^{-1} \mathrm{Na}_{2} \mathrm{SO}_{4}$ aqueous solution 


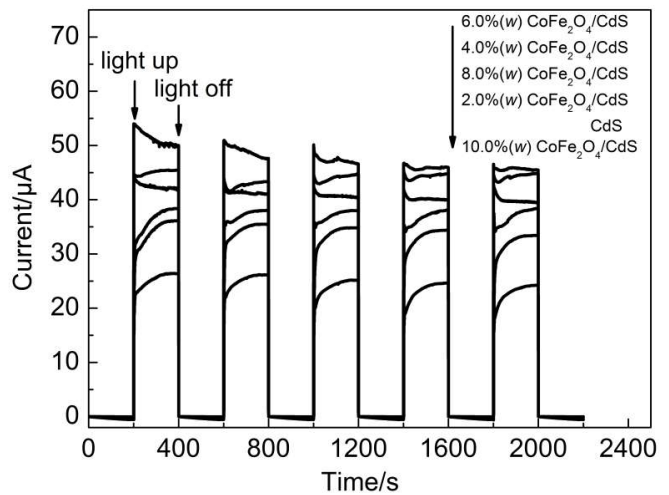

图 $7 \mathrm{CdS}$ 和 $p-\mathrm{CoFe}_{2} \mathrm{O}_{4} / n-\mathrm{CdS}$ 在 $0.1 \mathrm{~mol} \cdot \mathrm{L}^{-1} \mathrm{Na}_{2} \mathrm{SO}_{4}$ 溶液 中的瞬态光电流响应曲线

Fig.7 Transient photocurrent response of $\mathrm{CdS}$ and $p$ - $\mathrm{CoFe}_{2} \mathrm{O}_{4} / n-\mathrm{CdS}$ in $0.1 \mathrm{~mol} \cdot \mathrm{L}^{-1} \mathrm{Na}_{2} \mathrm{SO}_{4}$ aqueous solution

\section{2 $\mathrm{CoFe}_{2} \mathrm{O}_{4} / \mathrm{CdS}$ 光催化产氢性能}

\subsection{1 $\mathrm{CoFe}_{2} \mathrm{O}_{4}$ 含量的影响}

通过光催化产氢性能对 $\mathrm{CdS}$ 和 $p-\mathrm{CoFe}_{2} \mathrm{O}_{4} / n-$ $\mathrm{CdS}$ 的光催化活性进行评价。在 $35^{\circ} \mathrm{C}$ 下, 以 $\mathrm{CH}_{3} \mathrm{OH}\left(10 \%(\varphi), \mathrm{pH}\right.$ 为 6.0)为牺牲剂, $0.10 \mathrm{~g} \cdot \mathrm{L}^{-1}$ 光催化剂的产氢速率如图 8 所示。从图 8 可以看 出, 所有样品都具有光催化活性, $\mathrm{CoFe}_{2} \mathrm{O}_{4}$ 的含量 小于等于 $8.0 \%(w)$ 时, $p-\mathrm{CoFe}_{2} \mathrm{O}_{4} / n-\mathrm{CdS}$ 样品的产 氢速率大于 $\mathrm{CdS}$ 的产氢速率。随着 $\mathrm{CoFe}_{2} \mathrm{O}_{4}$ 含量的 增加, 产氢速率呈先增加后减小的趋势, $\mathrm{CoFe}_{2} \mathrm{O}_{4}$ 的含量为 $6.0 \%(w)$ 时, 产氢速率达到最大。

影响光催化剂产氢速率的因素有很多, 其中 光生电子-空穴对的分离效率是影响光催化反应的 关键因素。瞬态光电流响应曲线是分析电子-空穴 对分离效率的一种有效工具 ${ }^{28,29}$ 。由图 7 可知, 光 电流大小顺序为 $6.0 \%(w) \mathrm{CoFe}_{2} \mathrm{O}_{4} / \mathrm{CdS}>4.0 \%(w)$ $\mathrm{CoFe}_{2} \mathrm{O}_{4} / \mathrm{CdS}>8.0 \%$ (w) $\mathrm{CoFe}_{2} \mathrm{O}_{4} / \mathrm{CdS}>2.0 \%(w)$

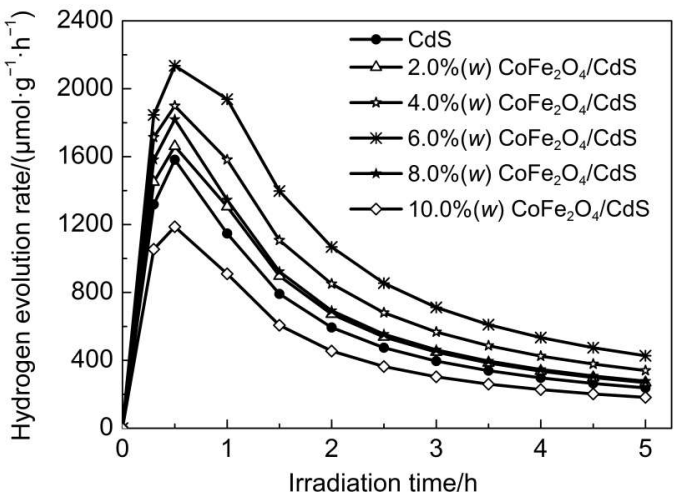

图 $8 \mathrm{CdS}$ 和 $p-\mathrm{CoFe}_{2} \mathrm{O}_{4} / n-\mathrm{CdS}\left(0.10 \mathrm{~g} \cdot \mathrm{L}^{-1}\right)$ 在 $\mathrm{CH}_{3} \mathrm{OH}$ 溶液中的可见光产氢速率图

Fig.8 Hydrogen evolution rate over $\mathrm{CdS}$ and $p$ - $\mathrm{CoFe}_{2} \mathrm{O}_{4} / n-\mathrm{CdS}\left(0.10 \mathrm{~g} \cdot \mathrm{L}^{-1}\right)$ under visible light irradiation in $\mathrm{CH}_{3} \mathrm{OH}$ solution

$\mathrm{CH}_{3} \mathrm{OH}$ initial volume fraction: $10 \%, \mathrm{pH}: 6.0$, reaction temperature: $35^{\circ} \mathrm{C}$

$\mathrm{CoFe}_{2} \mathrm{O}_{4} / \mathrm{CdS}>\mathrm{CdS}>10.0 \%(w) \mathrm{CoFe}_{2} \mathrm{O}_{4} / \mathrm{CdS}$ ， 与 产氢速率的大小顺序一致, 说明不同光催化剂产 氢速率的变化主要是光生电子-空穴对的分离效率 不同引起的。

$p-\mathrm{CoFe}_{2} \mathrm{O}_{4} / n-\mathrm{CdS}$ 的产氢速率比 $\mathrm{CdS}$ 的产氢速 率高, 主要原因有四个: 第一, $\mathrm{CoFe}_{2} \mathrm{O}_{4}$ 和 $\mathrm{CdS}$ 均 为窄带隙半导体, 二者复合后能增加对光的吸收 率, 提高了光催化活性; 第二, $\mathrm{CoFe}_{2} \mathrm{O}_{4}$ 的导带电 位比 $\mathrm{CdS}$ 的导带电位更负、价带电位比 $\mathrm{CdS}$ 的价带 电位更正, 二者复合以后, 产生能带交迭, 光生 电子可以从 $\mathrm{CoFe}_{2} \mathrm{O}_{4}$ 迁移到 $\mathrm{CdS}$, 光生空穴则可以 从 $\mathrm{CdS}$ 转移到 $\mathrm{CoFe}_{2} \mathrm{O}_{4}$, 如图 9 所示, 有效地抑制 了光生电子-空穴对的复合, 从而提高了光生载流 子的分离效率, 增强了光催化活性; 第三, $\mathrm{CoFe}_{2} \mathrm{O}_{4}$ 为 $p$ 型半导体, $\mathrm{CdS}$ 为 $n$ 型半导体，当 $p$ 型
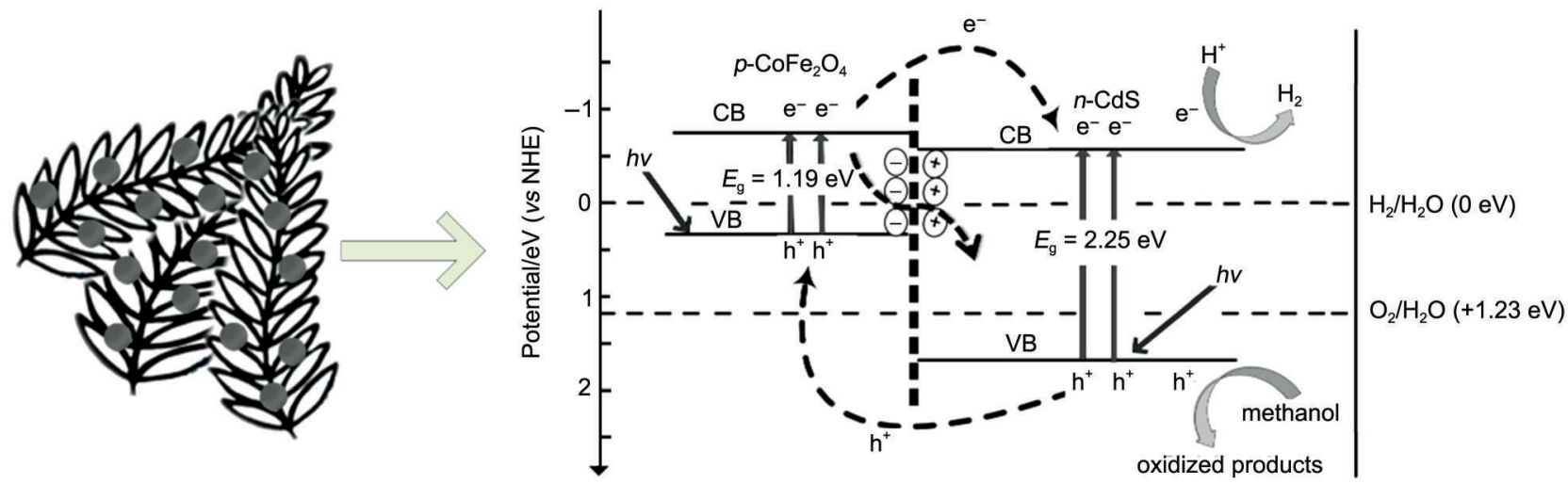

图 $9 p-\mathrm{CoFe}_{2} \mathrm{O}_{4} / n-\mathrm{CdS}$ 光催化剂的电荷迁移示意图

Fig.9 Transfer of charge carriers in the $p-\mathrm{CoFe}_{2} \mathrm{O}_{4} / n-\mathrm{CdS}$ photocatalyst NHE: normal hydrogen electrode; $\mathrm{CB}$ : conduction band; VB: valence band 
半导体 $\mathrm{CoFe}_{2} \mathrm{O}_{4}$ 和 $n$ 型半导体 $\mathrm{CdS}$ 复合时, 在 $\mathrm{CoFe}_{2} \mathrm{O}_{4}$ 和 $\mathrm{CdS}$ 的界面处会产生 $p-n$ 结, 如图 3 所 示, 在 $p-n$ 结内存在的内建电场的作用下, $p$ 型半 导体 $\mathrm{CoFe}_{2} \mathrm{O}_{4}$ 中的光生电子可以穿过 $p-n$ 结进入 $n$ 型 半导体 $\mathrm{CdS}$, 如图 9 所示, 光生空穴则可以从 $n$ 型 半导体 $\mathrm{CdS}$ 转移到 $p$ 型半导体 $\mathrm{CoFe}_{2} \mathrm{O}_{4}$ 上, 因而进 一步促进了光生电子-空穴对的迅速分离, 有效减 少光生电子-空穴对复合的概率, 从而进一步提高 了光催化活性。第四, $\mathrm{CdS}$ 独特的树形结构, 不仅 减少了自身团聚的现象, 增加了受辐射面积, 而 且增加了 $\mathrm{CoFe}_{2} \mathrm{O}_{4}$ 和 $\mathrm{CdS}$ 的接触面积, 即有效提高 了受辐照层 $p-n$ 结面积, 使得更多的光生电子在内 建电场的作用下从 $\mathrm{CoFe}_{2} \mathrm{O}_{4}$ 向 $\mathrm{CdS}$ 迁移, 减少了电 子-空穴对复合的概率。因此, $p$ - $\mathrm{CoFe}_{2} \mathrm{O}_{4} / n-\mathrm{CdS}$ 的 产氢速率大于 $\mathrm{CdS}$ 的产氢速率, 且随着 $\mathrm{CoFe}_{2} \mathrm{O}_{4}$ 含 量的增加, 产氢速率增加。当 $\mathrm{CoFe}_{2} \mathrm{O}_{4}$ 质量百分比 大于 $6.0 \%(w)$ 时, 产氢速率下降, 这可能是因为大 量的 $\mathrm{CoFe}_{2} \mathrm{O}_{4}$ 会成为光生电子与空穴复合的中心, 导致光催化活性的降低。

由图 8 还可以看出, 在反应的初始阶段, 产氢 速率逐渐上升, $0.5 \mathrm{~h}$ 后产氢速率达到最高值。此 后产氢速率迅速下降, 反应 $2 \mathrm{~h}$ 后, 产氢速率下降 缓慢。大部分文献认为 $\mathrm{CH}_{3} \mathrm{OH}$ 水溶液中光催化反 应的机制为 ${ }^{29}$ : 当光催化剂被可见光激发产生电 子-空穴对, 价带上的空穴会和吸附在光催化剂表面 的水分子作用生成 $\mathrm{H}^{+}$和 $\cdot \mathrm{OH}$, 生成的空穴及 $\cdot \mathrm{OH}$ 与 甲醛分子作用生成 $\mathrm{HCOOH}$ 和 $\mathrm{H}^{+}, \mathrm{HCOOH}$ 则进一 步被空穴氧化成 $\mathrm{CO}_{2}$ 和 $\mathrm{H}^{+}$, 电子则与生成的 $\mathrm{H}^{+}$反 应生成 $\mathrm{H}_{2}$ 。然而, 由于空穴 $\mathrm{h}^{+}$将水氧化成强氧化 剂 $\cdot \mathrm{OH}$ 的电位为 $E^{\circ}\left(\mathrm{H}_{2} \mathrm{O} / \cdot \mathrm{OH}\right)=+1.99 \mathrm{eV}(\mathrm{vs}$ $\mathrm{NHE})^{30-32}$, 而 $\mathrm{CoFe}_{2} \mathrm{O}_{4}$ 和 $\mathrm{CdS}$ 的价带电位值均小于 $1.99 \mathrm{eV}$, 故 $\mathrm{CoFe}_{2} \mathrm{O}_{4}$ 和 $\mathrm{CdS}$ 价带上的空穴不能将水 氧化生成强氧化剂· $\mathrm{OH}$, 这表明在此光催化过程 中, 氧化剂主要是空穴 $\mathrm{h}^{+}$。由于反应一段时间后光 催化反应液中检测到了中间体 $\mathrm{HCOOH}$ 的存在, 故 推测 $\mathrm{CH}_{3} \mathrm{OH}$ 水溶液中 $p-\mathrm{CoFe}_{2} \mathrm{O}_{4} / n-\mathrm{CdS}$ 为光催化剂 的光催化反应机制为 ${ }^{33}$ :

$$
\begin{aligned}
& \text { 光催化剂 }+h v \rightarrow \mathrm{e}^{-}+\mathrm{h}^{+} \\
& \mathrm{h}^{+}+\mathrm{e}^{-} \rightarrow \text { 热量 } \\
& \mathrm{CH}_{3} \mathrm{OH}+2 \mathrm{~h}^{+} \rightarrow \mathrm{HCHO}+2 \mathrm{H}^{+} \\
& \mathrm{HCHO}+\mathrm{H}_{2} \mathrm{O}+2 \mathrm{~h}^{+} \rightarrow \mathrm{HCOOH}+2 \mathrm{H}^{+} \\
& \mathrm{HCOOH} \rightarrow \mathrm{HCOO}^{-}+\mathrm{H}^{+} \\
& \mathrm{HCOO}^{-}+\mathrm{h}^{+} \rightarrow \cdot \mathrm{CO}_{2}^{-}+\mathrm{H}^{+}
\end{aligned}
$$

$$
\begin{aligned}
& \cdot \mathrm{CO}_{2}^{-}+\mathrm{h}^{+} \rightarrow \mathrm{CO}_{2} \\
& 2 \mathrm{H}^{+}+2 \mathrm{e}^{-} \rightarrow \mathrm{H}_{2}
\end{aligned}
$$

产氢速率随时间的延长呈现先增加后减少的 趋势, 是因为反应 $0.5 \mathrm{~h}$ 内, 随着反应时间的延 长, 越来越多的 $\mathrm{CH}_{3} \mathrm{OH}$ 作为牺牲剂被空穴氧化, 避免了光生电子-空穴对的复合, 使得越来越多的 $\mathrm{H}^{+}$能与光生电子反应生成 $\mathrm{H}_{2}$, 故产氢速率增加。 反应 $0.5 \mathrm{~h}$ 以后产氢速率开始下降, 这是因为由图 5 可知, 光催化剂的 $\mathrm{pH}_{\mathrm{prc}}$ 高于 $\mathrm{CH}_{3} \mathrm{OH}$ 水溶液的 $\mathrm{pH}$ 值 6.0 , 即光催化剂表面容易吸附负离子 ${ }^{13}$ 。因此, 根 据反应机理, 随着反应的进行, 越来越多的 $\mathrm{HCOO}^{-}$吸附在光催化剂表面, 若 $\mathrm{HCOO}^{-}$的生成速 率高于其消耗速率, 则过多的 $\mathrm{HCOO}^{-}$吸附在光催 化剂表面, 阻碍了 $\mathrm{CH}_{3} \mathrm{OH}$ 在光催化剂表面的吸 附, 从而使得单位时间内 $\mathrm{CH}_{3} \mathrm{OH}$ 与空穴接触的概 率降低, 则空穴与电子复合的概率增加, 产氢速 率下降。随着反应时间的进一步延长, 吸附在光 催化剂表面的 $\mathrm{HCOO}^{-}$逐渐被消耗, 光催化剂表面 重新裸露。反应 $2 \mathrm{~h}$ 以后, $\mathrm{HCOO}^{-}$在光催化剂表面 的吸附和消耗趋于稳定, 但是, 随着反应的进 行, 甲醇不断地被消耗, 抑制光生电子-空穴对复 合的能力下降, 因此, 反应 $2 \mathrm{~h}$ 以后产氢速率随着 时间延长缓慢下降。

\subsection{2 $\mathrm{CH}_{3} \mathrm{OH}$ 浓度对光催化制氢性能的影响}

反应温度 $35{ }^{\circ} \mathrm{C}$, 光催化剂 $6.0 \%(w) \mathrm{CoFe}_{2} \mathrm{O}_{4} /$ $\mathrm{CdS}$ 的用量为 $0.10 \mathrm{~g} \cdot \mathrm{L}^{-1}$, 探索了 $\mathrm{CH}_{3} \mathrm{OH}$ 浓度 $(\mathrm{pH}$ 为 6.0)对光催化分解水制氢性能的影响。图 10 为 产氢速率图。从图中还可以看出: 随着 $\mathrm{CH}_{3} \mathrm{OH}$ 浓 度的增加, 产氢速率呈先增加后减少的趋势。当 $\mathrm{CH}_{3} \mathrm{OH}$ 的浓度为 $10 \%(\varphi$, 体积分数) 时, 产氢速率 最大。

根据 3.2.1 节给出的反应机理, $\mathrm{CH}_{3} \mathrm{OH}$ 含量较 低时, 吸附在光催化剂表面上的 $\mathrm{CH}_{3} \mathrm{OH}$ 量较少, 抑制光生电子-空穴对复合的作用不明显, 所以产 氢速率较低。随着 $\mathrm{CH}_{3} \mathrm{OH}$ 含量的增加, 越来越多 的光生空穴被消耗掉生成 $\mathrm{H}^{+}$, 使得更多的光生电 子可以按式 $(8)$ 反应生成 $\mathrm{H}_{2}$, 产氢速率增加。但 是, 随着 $\mathrm{CH}_{3} \mathrm{OH}$ 浓度的进一步增加, 产氢速率下 降, 这是因为中间产物 $\mathrm{HCOO}^{-}$吸附在光催化剂的 表面, 阻碍了 $\mathrm{CH}_{3} \mathrm{OH}$ 在光催化剂表面的吸附, 所 以导致产氢速率下降。

由图 10 还可以看出, $\mathrm{CH}_{3} \mathrm{OH}$ 初始浓度不同 时, 产氢速率随时间的延长同样呈现先增加后减 

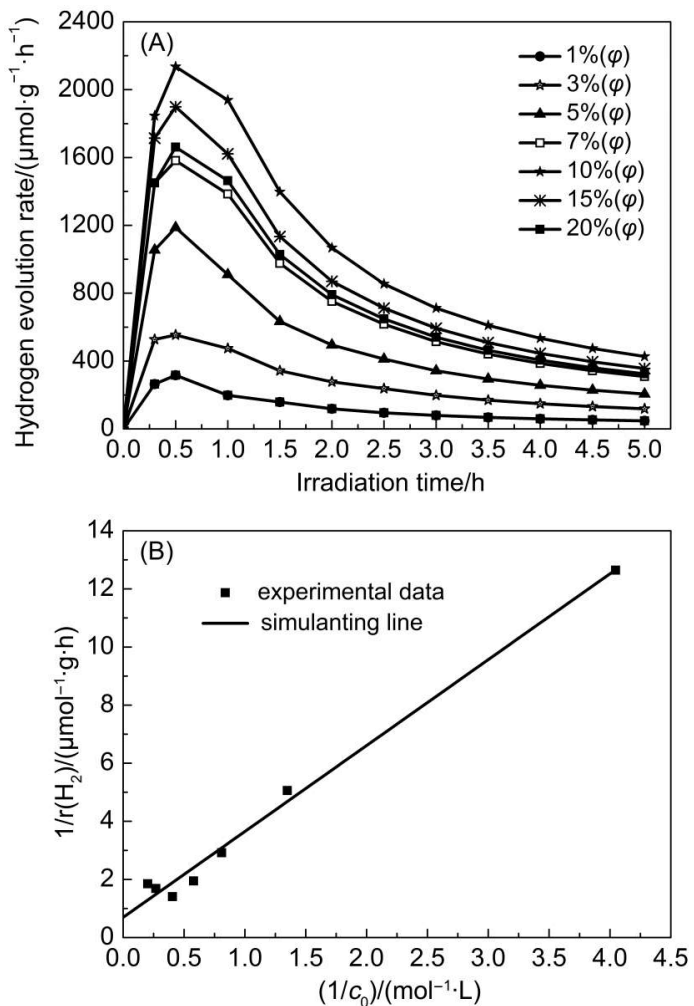

图 10 (A) $\mathrm{CH}_{3} \mathrm{OH}$ 浓度 $(\mathrm{pH}=6.0)$ 对光催化产氢速率的影响 $\left(0.10 \mathrm{~g} \cdot \mathrm{L}^{-1}\right.$ 的 $6.0 \%(w) \mathrm{CoFe}_{2} \mathrm{O}_{4} / \mathrm{CdS}$, 反应温度 $\left.35^{\circ} \mathrm{C}\right)$;

(B) $1 / r\left(\mathrm{H}_{2}\right)$ 与 $1 / c_{0}$ 关系曲线

Fig.10 (A) Effect of the amount of $\mathrm{CH}_{3} \mathrm{OH}(\mathrm{pH}=6.0)$ on photocatalytic hydrogen evolution rates $\left(0.10 \mathrm{~g} \cdot \mathrm{L}^{-1}\right.$ of $6.0 \%$ (w) $\mathrm{CoFe}_{2} \mathrm{O}_{4} / \mathrm{CdS}$, reaction temperature $35^{\circ} \mathrm{C}$ );

(B) plot of $1 / r\left(\mathrm{H}_{2}\right)$ versus $1 / c_{0}$

$r\left(\mathrm{H}_{2}\right)$ : hydrogen evolution rate, $c_{0}$ : initial concentration of $\mathrm{CH}_{3} \mathrm{OH}$

少的趋势。这同样是因为反应的初始阶段, $\mathrm{CH}_{3} \mathrm{OH}$ 作为牺牲剂消耗了空穴, 使得越来越多的 $\mathrm{H}^{+}$能与光生电子反应生成 $\mathrm{H}_{2}$, 故产氢速率增加。 随着反应的进行, 越来越多的中间产物 $\mathrm{HCOO}^{-}$吸 附在光催化剂表面, 阻碍了 $\mathrm{CH}_{3} \mathrm{OH}$ 在光催化剂表 面的吸附，导致产氢速率下降，反应 $2 \mathrm{~h}$ 以后，产 氢速率趋于稳定, 并随着甲醇的不断消耗而缓慢 下降。

光催化反应属于多相界面反应过程, 产氢速 率可表示为 ${ }^{34}$ :

$$
r\left(\mathrm{H}_{2}\right)=\frac{\mathrm{d} n\left(\mathrm{H}_{2}\right)}{\mathrm{d} t}=\frac{k\left(\mathrm{H}_{2}\right) K c_{0}}{1+K c_{0}}
$$

式中 $r\left(\mathrm{H}_{2}\right)$ 是起始时的产氢速率, $\mathrm{mmol} \cdot \mathrm{g}^{-1} \cdot \mathrm{h}^{-1}$; $n\left(\mathrm{H}_{2}\right)$ 为单位质量的催化剂产生的氢气的物质的 量, $\mathrm{mmol} \cdot \mathrm{g}^{-1} ; c_{0}$ 是 $\mathrm{CH}_{3} \mathrm{OH}$ 的起始浓度, $\mathrm{mol} \cdot$ $\mathrm{L}^{-1} ; k\left(\mathrm{H}_{2}\right)$ 是产氢速率常数, $\mathrm{mmol} \cdot \mathrm{g}^{-1} \cdot \mathrm{h}^{-1} ; K$ 是 $\mathrm{CH}_{3} \mathrm{OH}$ 在 $6.0 \%(w) \mathrm{CoFe}_{2} \mathrm{O}_{4} / \mathrm{CdS}$ 光催化剂上吸附常 数, $\mathrm{L} \cdot \mathrm{mol}^{-1}$; 为了确定 $k\left(\mathrm{H}_{2}\right)$ 和 $K$ 的值, 式(9)可以
写成式(10):

$$
\frac{1}{r\left(\mathrm{H}_{2}\right)}=\frac{1}{k\left(\mathrm{H}_{2}\right)}+\frac{1}{K k\left(\mathrm{H}_{2}\right)} \cdot \frac{1}{c_{0}}
$$

以 $1 / r\left(\mathrm{H}_{2}\right)$ 对 $1 / c_{0}$ 作图, 结果如图 10(B) 所示, 对 $1 / r\left(\mathrm{H}_{2}\right)-1 / c_{0}$ 曲线的数据进行线性回归, 得到一 条直线(相关系数 $R=0.988)$ 。由式(10)知, 直线的 斜率为 $1 / K k\left(\mathrm{H}_{2}\right)$, 直线的截距为 $1 / k\left(\mathrm{H}_{2}\right)$ 。计算得到 产氢速率常数 $k\left(\mathrm{H}_{2}\right)$ 为 $1.44 \mathrm{mmol} \cdot \mathrm{g}^{-1} \cdot \mathrm{h}^{-1}, \mathrm{CH}_{3} \mathrm{OH}$ 在光催化剂表面吸附常数 $K$ 为 $0.162 \mathrm{~L} \cdot \mathrm{mol}^{-1}$ 。

\subsection{3 溶液 $\mathrm{pH}$ 的影响}

$0.10 \mathrm{~g} \cdot \mathrm{L}^{-1}$ 的 $6.0 \%(w) \mathrm{CoFe}_{2} \mathrm{O}_{4} / \mathrm{CdS}$ 为光催化 剂, $\mathrm{CH}_{3} \mathrm{OH}(10 \%(\varphi))$ 为牺牲剂, 用 $0.1 \mathrm{~mol} \cdot \mathrm{L}^{-1}$ 的 盐酸和 $0.1 \mathrm{~mol} \cdot \mathrm{L}^{-1}$ 氢氧化钠调节 $\mathrm{pH}$, 探索了溶液 $\mathrm{pH}$ 对的可见光催化制氢性能的影响。图 11(A)为产 氢速率图。

由图 11(A) 可以看出, $\mathrm{CH}_{3} \mathrm{OH}$ 水溶液的 $\mathrm{pH}$ 对 产氢速率有很大的影响。随着 $\mathrm{CH}_{3} \mathrm{OH}$ 水溶液 $\mathrm{pH}$ 的 增加, 产氢速率呈先增加后减少的趋势。当 $\mathrm{pH}$ 为 6 时, 产氢速率最大。图 11(B)为暗反应时, 溶液
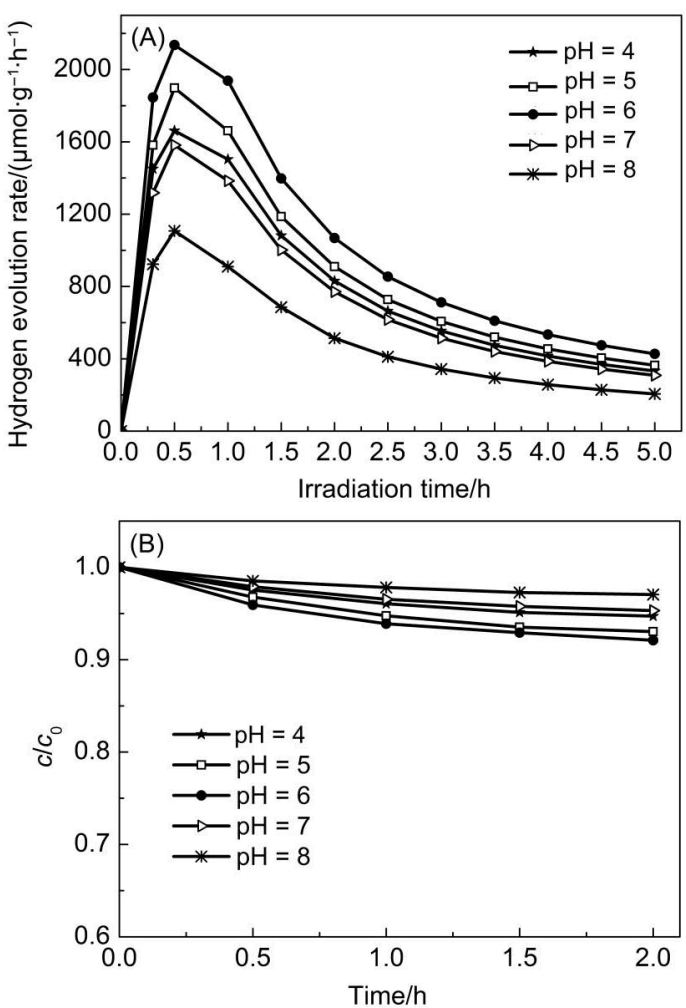

图 11 (A) pH 对产氢速率的影响 $\left(0.10 \mathrm{~g} \cdot \mathrm{L}^{-1}\right.$ 的 $6.0 \%(w)$ $\mathrm{CoFe}_{2} \mathrm{O}_{4} / \mathrm{CdS}, 10 \%(\varphi)$ 的 $\left.\mathrm{CH}_{3} \mathrm{OH}\right)$; (B) $\mathrm{pH}$ 对 $6.0 \%(w)$ $\mathrm{CoFe}_{2} \mathrm{O}_{4} / \mathrm{CdS}\left(0.1 \mathrm{~g} \cdot \mathrm{L}^{-1}\right)$ 吸附 $10 \%(\varphi) \mathrm{CH}_{3} \mathrm{OH}$ 性能的影响 Fig.11 (A) Effects of $\mathbf{p H}$ value on photocatalytic hydrogen evolution rate $\left(0.10 \mathrm{~g} \cdot \mathrm{L}^{-1}\right.$ of $6.0 \%(w) \mathrm{CoFe}_{2} \mathrm{O}_{4} / \mathrm{CdS}, 10 \%$ $(\varphi)$ of $\mathrm{CH}_{3} \mathrm{OH}$ ); (B) effects of $\mathrm{pH}$ value on the adsorption of $10 \%(\varphi) \mathrm{CH}_{3} \mathrm{OH}$ over $6.0 \%(w) \mathrm{CoFe}_{2} \mathrm{O}_{4} / \mathrm{CdS}\left(0.1 \mathrm{~g} \cdot \mathrm{L}^{-1}\right)$ 
$\mathrm{pH}$ 对 $6.0 \%(w) \mathrm{CoFe}_{2} \mathrm{O}_{4} / \mathrm{CdS}\left(0.1 \mathrm{~g} \cdot \mathrm{L}^{-1}\right)$ 吸附 $10 \%(\varphi)$ 甲醇性能的影响。甲醇浓度采用 FULI 9790 气相色 谱仪进行检测, 检测条件为: 填充柱中固定相为 Porapak Q, $\mathrm{H}_{2}$ 作载气, $\mathrm{H}_{2}$ 流速为 $35 \mathrm{~mL} \cdot \mathrm{min}^{-1}$, 热 导检测器, 柱温 $120^{\circ} \mathrm{C}$, 汽化温度 $120^{\circ} \mathrm{C}$, 检测温 度 $120^{\circ} \mathrm{C}$ 。从图 $11(\mathrm{~B})$ 可以看出, 溶液 $\mathrm{pH}$ 不同时, 甲醇在 $6.0 \%(w) \mathrm{CoFe}_{2} \mathrm{O}_{4} / \mathrm{CdS}$ 表面吸附量大小的顺 序是: $\mathrm{pH}=6$ 的吸附量 $>\mathrm{pH}=5$ 的吸附量 $>\mathrm{pH}=4$ 的吸附量 $>\mathrm{pH}=7$ 的吸附量 $>\mathrm{pH}=8$ 的吸附量, 与 产氢速率的大小顺序一致。图 12 为不同 $\mathrm{pH}$ 值下 $6.0 \%$ (w) $\mathrm{CoFe}_{2} \mathrm{O}_{4} / \mathrm{CdS}$ 的瞬态光电流, 由图可知, 随着 $\mathrm{pH}$ 值的增加, 光电流值增加, 说明光生电子空穴对的分离效率增加, 光催化活性增强。由此 可见, 甲醇分子与光催化剂之间吸附作用的强弱 对产氢速率有重要影响。当 $\mathrm{pH}<7$ 时, 随着 $\mathrm{pH}$ 值 的增加, 甲醇在光催化剂表面吸附量增加, 光电 流值也增加, 光催化活性增强, 且此时溶液的 $\mathrm{pH}$ 值低于光催化剂的 $\mathrm{pH}_{\mathrm{prc}}$, 即光催化剂表面容易吸 附负离子 $\mathrm{HCOO}^{-}$, 吸附的 $\mathrm{HCOO}^{-}$被空穴还原成 $\mathrm{H}^{+}$, 有利于 (8) 式反应的进行, 因此产氢速率增 加。产氢速率随时间的延长同样呈现先增加后减 少的趋势。这同样是因为反应的初始阶段, $\mathrm{CH}_{3} \mathrm{OH}$ 消耗了空穴, 提高了产氢速率。随着反应 的进行, 光催化剂表面 $\mathrm{HCOO}^{-}$的吸附阻碍了 $\mathrm{CH}_{3} \mathrm{OH}$ 的吸附, 导致产氢速率下降, 反应 $2 \mathrm{~h}$ 以 后, 产氢速率趋于稳定, 并随着甲醇的不断消耗 而缓慢下降。当 $\mathrm{pH} \geq 7$ 时, 虽然随着 $\mathrm{pH}$ 值的增 加, 光电流值增加, 光生电子-空穴对的分离效率 增加, 但是甲醇在光催化剂表面吸附量降低, 不 利于消耗空穴, 此外, 由图 4 可知, 溶液的 $\mathrm{pH}$ 值

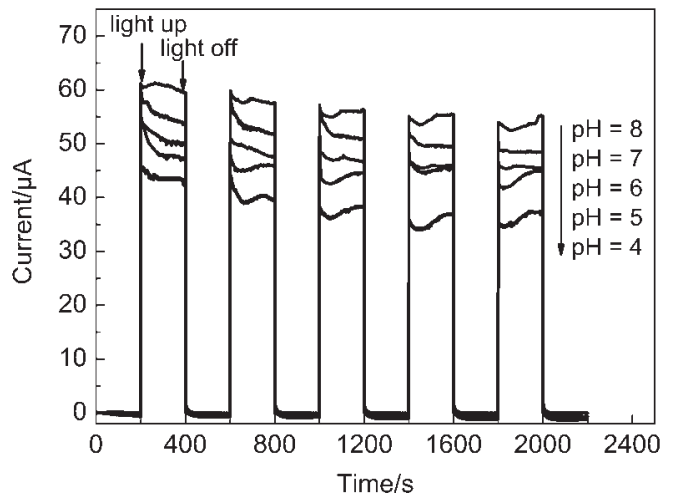

图 12 不同 $\mathrm{pH}$ 值下 $6.0 \%(w) \mathrm{CoFe}_{2} \mathrm{O}_{4} / \mathrm{CdS}$ 的瞬态光电流 Fig.12 Transient photocurrent of $6.0 \%(w) \mathrm{CoFe}_{2} \mathrm{O}_{4} / \mathrm{CdS}$ at different $\mathrm{pH}$ values
高于光催化剂的 $\mathrm{pH}_{\mathrm{pzc}}$, 即光催化剂表面不容易吸 附负离子, 不利于产生 $\mathrm{H}^{+}$的反应进行, 产氢速率 下降。因此, $\mathrm{CH}_{3} \mathrm{OH}$ 水溶液的最佳 $\mathrm{pH}$ 值为 6.0 , 是 $\mathrm{pH}$ 影响光催化剂中光生电子-空穴对的分离效率 以及光催化剂表面吸附性能的综合体现。当 $\mathrm{pH} \geq$ 7 时, 产氢速率随时间的延长依然呈现先增加后减 少的趋势。反应的初始阶段, $\mathrm{CH}_{3} \mathrm{OH}$ 消耗了空 穴, 提高了产氢速率。由于溶液的 $\mathrm{pH}$ 值高于光催 化剂的 $\mathrm{pH}_{\mathrm{prc}}$, 光催化剂表面容易吸附正离子。因 此, 随着反应的进行, 越来越多的 $\mathrm{H}^{+}$吸附在光催 化剂表面, 同样阻碍了 $\mathrm{CH}_{3} \mathrm{OH}$ 在光催化剂表面的 吸附, 导致产氢速率下降, 反应 $2 \mathrm{~h}$ 以后, 产氢速 率趋于稳定, 并随着甲醇的不断消耗而缓慢下降。

\subsection{4 光催化剂用量的影响}

反应温度 $35^{\circ} \mathrm{C}, \mathrm{CH}_{3} \mathrm{OH}(10 \%(\varphi), \mathrm{pH}$ 为 6.0$)$ 为牺牲剂, 探索了复合光催化剂 $6.0 \%(w) \mathrm{CoFe}_{2} \mathrm{O}_{4} /$ $\mathrm{CdS}$ 的用量对可见光催化制氢性能的影响。图 13 为 $5 \mathrm{~h}$ 内的平均产氢速率图。

从图可以很明显看出, 随着光催化剂用量的 增加, 产氢量逐渐增加。当光催化剂用量为 0.10 $\mathrm{g} \cdot \mathrm{L}^{-1}$ 时, 平均产氢速率达到最大。随着光催化剂 用量的进一步增加, 产氢速率降低。这是因为增 加光催化剂用量可以增加光催化剂表面对光的吸 收率, 从而增加产氢速率。但是, 较大的光催化 剂用量会增加溶液的浊度, 导致光催化剂对光的 散射作用增强, 可见光的渗透性降低, 被光激发 的光催化剂的量反而下降, 从而使得光催化活性 降低 ${ }^{35}$ 。

\section{3 光催化剂的稳定性}

$35{ }^{\circ} \mathrm{C}$ 下, 以 $\mathrm{CH}_{3} \mathrm{OH}(10 \%(\varphi), \mathrm{pH}$ 为 6.0)为牺

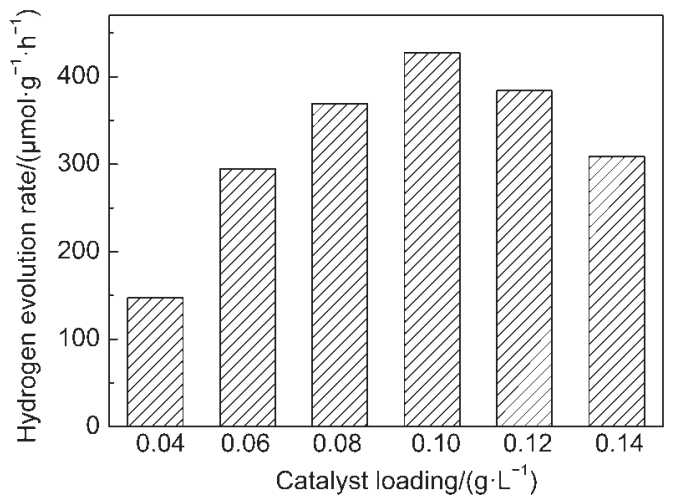

图 13 光催化剂用量对光催化产氢速率的影响

Fig.13 Effect of photocatalyst loading on photocatalytic hydrogen evolution rate

$10 \%(\varphi) \mathrm{CH}_{3} \mathrm{OH}$, reaction temperature: $35^{\circ} \mathrm{C}$, reaction time: $5 \mathrm{~h}$ 
牲剂, 分别用 $0.1 \mathrm{~g} \cdot \mathrm{L}^{-1}$ 的 $6.0 \%(w) \mathrm{CoFe}_{2} \mathrm{O}_{4} / \mathrm{CdS}$ 和 $\mathrm{CdS}$ 为光催化剂, 探讨光催化剂的稳定性。每次光 照反应 $5 \mathrm{~h}$, 连续进行光催化反应 5 次, 每次在 $5 \mathrm{~h}$ 内的平均产氢速率如图 14 所示。由图 14 可见: $6.0 \%(w) \mathrm{CoFe}_{2} \mathrm{O}_{4} / \mathrm{CdS}$ 第 5 次反应的产氢速率是初 次反应的产氢速率的 $87.86 \%, \mathrm{CdS}$ 第 5 次反应的产 氢速率是初次反应的产氢速率的 $80.72 \%$ 。由此可 见, 与 $\mathrm{CdS}$ 相比, $6.0 \%(w) \mathrm{CoFe}_{2} \mathrm{O}_{4} / \mathrm{CdS}$ 具有较好 的光稳定性，在循环使用 5 次后，依然保持较高的 光催化活性。

为了探讨 $6.0 \%(w) \mathrm{CoFe}_{2} \mathrm{O}_{4} / \mathrm{CdS}$ 和 $\mathrm{CdS}$ 的光腐 蚀行为, 以 $350 \mathrm{~W}$ 氙灯为光源, 分别将 $0.1 \mathrm{~g}$ 的 $6.0 \%(w) \mathrm{CoFe}_{2} \mathrm{O}_{4} / \mathrm{CdS}$ 和 $\mathrm{CdS}$ 置于 $100 \mathrm{~mL}$ 去离子水 中, 在磁力搅拌下进行光照, 每隔一段时间取悬 浮液离心分离, 采用 OPTIMA 2000DV 型等离子发 射光谱仪(美国 PE 公司)测量上层清液中 $\mathrm{Cd}^{2+}$ 的浓 度。为了比较牺牲剂对 $\mathrm{CdS}$ 光腐蚀的抑制作用, 将 $0.1 \mathrm{~g}$ 的 $6.0 \%(w) \mathrm{CoFe}_{2} \mathrm{O}_{4} / \mathrm{CdS}$ 置于 $100 \mathrm{~mL}$ 的 $\mathrm{CH}_{3} \mathrm{OH}$ 水溶液 $(10 \%(\varphi), \mathrm{pH}$ 为 6.0) 中, 在磁力搅拌 下进行光照, 每隔一段时间取悬浮液离心分离, 去 $\mathrm{CH}_{3} \mathrm{OH}$ 后, 测量上层清液中 $\mathrm{Cd}^{2+}$ 的浓度。反应 体系中 $\mathrm{Cd}^{2+}$ 浓度的变化如图 15 所示。

由图 15 可以看出, 当 $6.0 \%(w) \mathrm{CoFe}_{2} \mathrm{O}_{4} / \mathrm{CdS}$ 和 $\mathrm{CdS}$ 分别放在去离子水中时, $6.0 \%(w) \mathrm{CoFe}_{2} \mathrm{O}_{4} /$ $\mathrm{CdS}$ 悬浮液中 $\mathrm{Cd}^{2+}$ 的浓度小于 $\mathrm{CdS}$ 悬浮液中 $\mathrm{Cd}^{2+}$ 的 浓度。 $\mathrm{CdS}$ 的光腐蚀作用是指 $\mathrm{CdS}$ 和光生空穴反应 产生 $\mathrm{Cd}^{2+}$ 的过程。由于 $6.0 \%(w) \mathrm{CoFe}_{2} \mathrm{O}_{4} / \mathrm{CdS}$ 光催 化剂中通过能带交迭和 $p-n$ 结中存在的内建电场的 双重作用, 光生空穴可以从 $\mathrm{CdS}$ 转移到 $\mathrm{CoFe}_{2} \mathrm{O}_{4}$ 上, 减少了光生空穴和 $\mathrm{CdS}$ 反应生成 $\mathrm{Cd}^{2+}$ 的概率。

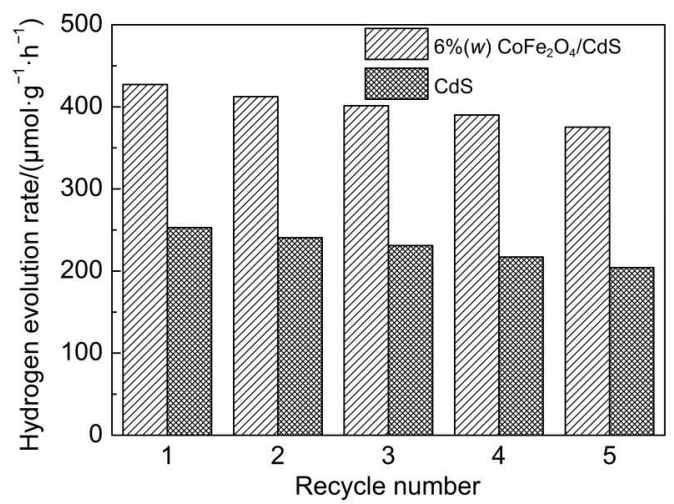

图 $146.0 \%(w) \mathrm{CoFe}_{2} \mathrm{O}_{4} / \mathrm{CdS}$ 和 $\mathrm{CdS}$ 的循环使用性能

Fig.14 Recycling performance of $6.0 \%(w) \mathrm{CoFe}_{2} \mathrm{O}_{4} / \mathrm{CdS}$ and $\mathrm{CdS}$

Each recycling time is $5 \mathrm{~h}$.

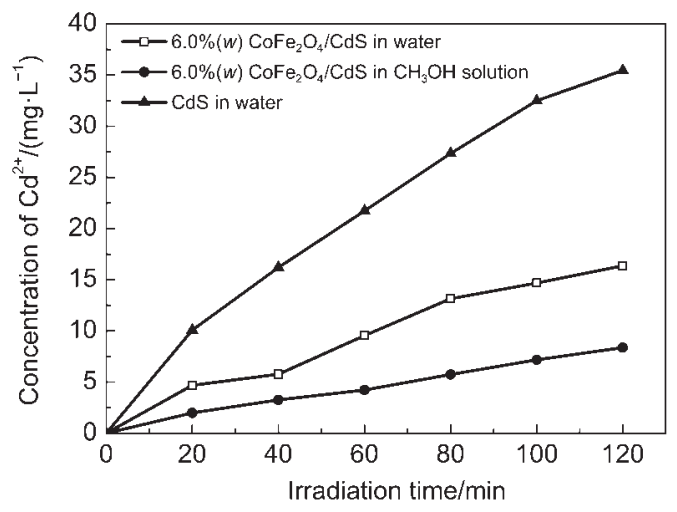

图 $15 \mathrm{Cd}^{2+}$ 的浓度与光照时间的关系

Fig.15 Relation between concentration of $\mathrm{Cd}^{2+}$ and irradiation time

因此, $6.0 \%(w) \mathrm{CoFe}_{2} \mathrm{O}_{4} / \mathrm{CdS}$ 悬浮液中 $\mathrm{Cd}^{2+}$ 的浓度 小于 $\mathrm{CdS}$ 悬浮液中 $\mathrm{Cd}^{2+}$ 的浓度。当 $6.0 \%(w)$ $\mathrm{CoFe}_{2} \mathrm{O}_{4} / \mathrm{CdS}$ 分别放在去离子水和 $\mathrm{CH}_{3} \mathrm{OH}$ 水溶液中 时, $\mathrm{CH}_{3} \mathrm{OH}$ 水溶液中 $\mathrm{Cd}^{2+}$ 的浓度小于水中 $\mathrm{Cd}^{2+}$ 的浓 度，说明制氢体系中牺牲剂甲醇的存在不仅有利 于消耗空穴, 避免电子-空穴对的复合, 提高光催 化活性，而且抑制了 $\mathrm{CdS}$ 的光腐蚀。从图 15 还可 以看出, $\mathrm{CoFe}_{2} \mathrm{O}_{4}$ 与 $\mathrm{CdS}$ 复合产生的能带交迭和内 建电场的双重作用是抑制 $\mathrm{CdS}$ 光腐蚀的主要原 因，其抑制效果好于甲醇对 $\mathrm{CdS}$ 光腐蚀的抑制效 果。综上所述, 图 14 所示的循环反应中, 产氢速 率的减少主要是循环反应过程中离心沉降等操作 造成光催化剂的损失的缘故。

\section{4 结 论}

用 $\mathrm{CdCl}_{2} \cdot x \mathrm{H}_{2} \mathrm{O} 、 \mathrm{CoCl}_{2} \cdot 6 \mathrm{H}_{2} \mathrm{O} 、 \mathrm{FeCl}_{3} \cdot 6 \mathrm{H}_{2} \mathrm{O}$ 、 $\mathrm{NaOH}$ 和 $\mathrm{CN}_{2} \mathrm{H}_{4} \mathrm{~S}$ 为原料, 采用水热法制备了系列 $p$ - $n$ 复合半导体 $p$ - $\mathrm{CoFe}_{2} \mathrm{O}_{4} / n-\mathrm{CdS}$ 。采用 $\mathrm{XRD}$ 、 SEM、UV-Vis DRS 和电化学工作站等对产物进行 了结构和性能表征。以 $\mathrm{CH}_{3} \mathrm{OH}$ 为牺牲剂, 用可见 光催化产氢速率对催化剂的光催化活性进行了评 价, 探讨了 $\mathrm{CoFe}_{2} \mathrm{O}_{4}$ 含量、 $\mathrm{CH}_{3} \mathrm{OH}$ 浓度、反应体系 $\mathrm{pH}$ 和光催化剂用量对产氢速率的影响, 并研究了 其光腐蚀行为。结果表明:

$\mathrm{CH}_{3} \mathrm{OH}$ 浓度为 $10 \%(\varphi)$ 、光催化剂用量为 0.1 $\mathrm{g} \cdot \mathrm{L}^{-1}$ 、反应体系 $\mathrm{pH}$ 为 6 时, $6.0 \%(w) p-\mathrm{CoFe}_{2} \mathrm{O}_{4} / n$ $\mathrm{CdS}$ 具有最高的光催化活性, 产氢速率常数 $k\left(\mathrm{H}_{2}\right)$ 为 $1.44 \mathrm{mmol} \cdot \mathrm{g}^{-1} \cdot \mathrm{h}^{-1}, \mathrm{CH}_{3} \mathrm{OH}$ 在光催化剂表面吸附 常数 $(K)$ 为 $0.162 \mathrm{~L} \cdot \mathrm{mol}^{-1}$;

$p-\mathrm{CoFe}_{2} \mathrm{O}_{4} / n-\mathrm{CdS}$ 光催化活性的提高是由于 $\mathrm{CoFe}_{2} \mathrm{O}_{4}$ 和 $\mathrm{CdS}$ 两种窄带隙半导体复合增加了光吸 
收率; $\mathrm{CoFe}_{2} \mathrm{O}_{4}$ 和 $\mathrm{CdS}$ 二者复合所产生的能带交迭 和内建电场的作用, 促进了电子从 $\mathrm{CoFe}_{2} \mathrm{O}_{4}$ 向 $\mathrm{CdS}$ 的迁移, 减少电子-空穴对复合的概率; $\mathrm{CdS}$ 独特 的树形结构, 不仅减少了自身团聚的现象, 增加 了受辐射面积, 而且增加了 $\mathrm{CoFe}_{2} \mathrm{O}_{4}$ 和 $\mathrm{CdS}$ 的接触 面积, 即有效提高了受辐照层 $p-n$ 结面积, 进一步 减少了电子-空穴对复合的概率的缘故;

光催化剂中光生电子-空穴对的分离效率, 以 及牺牲剂和反应中间产物与光催化剂之间吸附作 用的强弱都对产氢速率有重要影响;

$\mathrm{CH}_{3} \mathrm{OH}$ 水溶液的 $\mathrm{pH}$ 对产氢速率有很大影响, 是溶液 $\mathrm{pH}$ 影响光催化剂中光生电子-空穴对的分离 效率以及光催化剂表面吸附性能的综合体现;

光腐蚀行为研究结果表明, 制氢体系中牺牲 剂 $\mathrm{CH}_{3} \mathrm{OH}$ 的存在以及制备成 $p-n$ 复合半导体 $p$ $\mathrm{CoFe}_{2} \mathrm{O}_{4} / n-\mathrm{CdS}$, 均能抑制 $\mathrm{CdS}$ 的光腐蚀, 而 $p$ $\mathrm{CoFe}_{2} \mathrm{O}_{4} / n-\mathrm{CdS}$ 所产生的能带交迭和内建电场的双 重作用, 对 $\mathrm{CdS}$ 光腐蚀的抑制效果更好, 因此, $p$ $\mathrm{CoFe}_{2} \mathrm{O}_{4} / n-\mathrm{CdS}$ 的稳定性较好, 可多次循环使用。

\section{References}

(1) Guo, X. Y.; Chen, C. F.; Song, W. Y.; Wang, X.; Di, W. H.; Qin, W. P. J. Mol. Catal. A-Chem. 2014, 387, 1. doi: 10.1016/j. molcata.2014.02.020

(2) Yan, J. J.; Wang, K.; Xu, H.; Qian, J.; Liu, W.; Yang, X. W.; Li, H. M. Chin. J. Catal. 2013, 34 (10), 1876. doi: 10.1016/S18722067(12)60677-9

(3) Wang, C.; Wang, L.; Jin, J.; Liu, J.; Li, Y.; Wu, M.; Chen, L. H.; Wang, B. J.; Yang, X. Y.; Su, B. L. Appl. Catal. B 2016, 188, 351. doi: 10.1016/j.apcatb.2016.02.017

(4) Wang, Y. J.; Sun, J. Y.; Feng, R. J.; Zhang, J. Acta Phys. -Chim. Sin. 2016, 32 (3), 128. [王彦娟, 孙佳瑶, 封瑞江, 张 健. 物理 化学学报, 2016, 32 (3), 128.] doi: 10.3866/PKU. WHXB201511303

(5) Xing, W. N.; Ni, L.; Liu, X. L.; Luo, Y. Y.; Lu, Z. Y.; Yan, Y. S.; Huo, P. W. Desalin. Water Treat. 2015, 53 (3), 794. doi: 10.1080/ 19443994.2013.844082

(6) Yang, J. H.; Yan, H. J.; Wang, X. L.; Wen, F. Y.; Wang, Z. J.; Fan, D. Y.; Shi, J. Y.; Li, C. J. Catal. 2012, 290, 151. doi: 10.1016/j.jcat.2012.03.008

(7) Sasikala, R.; Shirole, A. R.; Sudarsan, V.; Girija, K. G.; Rao, R.; Sudakar, C.; Bharadwaj, S. R. J. Mater. Chem. 2011, 21 (41), 16566. doi: $10.1039 / \mathrm{c} 1 \mathrm{jm} 12531 \mathrm{a}$

(8) Xing, W. N.; Ni, L; Yan, X. S.; Liu, X. L.; Luo, Y. Y.; Lu, Z. Y.; Yan, Y. S.; Huo, P. W. Acta Phys. -Chim. Sin. 2014, 30 (1), 141. [邢伟男, 倪 良, 颜学升, 刘馨琳, 罗莹藏, 逯子扬, 问永胜, 霍 鹏伟. 物理化学学报, 2014, 30 (1), 141.] doi: 10.3866/PKU.
WHXB201311211

(9) Du, H.; Wang, S.; Liu, L. L.; Liu, Z. X.; Li, Z.; Lu, N.; Liu, F. S. Acta Phys. -Chim. Sin. 2010, 26 (10), 2726. [杜 欢, 王 星, 刘 恋恋, 刘忠祥, 李 振, 卢 南, 刘福生. 物理化学学报, 2010, 26 (10), 2726.] doi: 10.3866/PKU.WHXB20101023

(10) Liu, F. S.; Wang, S.; Liu, L. L.; Du, H. Adv. Mater. Res. 2012, 512, 1677. doi: 10.4028/www.scientific.net/AMR.512-515.1677

(11) An, L.; Wang, G. H.; Cheng, Y.; Zhao, L.; Gao, F.; Cheng, Y. Russ. J. Phys. Chem. A 2015, 89 (10), 1878. doi: 10.1134/ $\mathrm{S} 0036024415100180$

(12) Shen, J. F.; Huang, W. S.; Li, N.; Ye, M. X. Ceram. Int. 2014, 41 (1), 761. doi: 10.1016/j.ceramint.2014.08.135

(13) Zhang, D.; Liu, F. S.; Wang, S.; Li, Z.; Qian, Q. Q.; Wang, X. Q.; Si, G. L. Mater. Sci. Semicond. Process. 2015, 40, 602. doi: 10.1016/j.mssp.2015.06.043

(14) Ye, L. J.; Jiang, Y. J.; Yan, C.; An, X. Y.; Li, N. Appl. Chem. Industry. 2015, 44 (11), 1992. [叶林静, 姜韵婕, 间 超, 安小 英, 李 娜. 应用化工, 2015, 44 (11), 1992.] doi: 10.16581/j. cnki.issn1671-3206.2015.11.006

(15) Singh, S.; Khare, N. RSC Adv. 2015, 5 (117), 96562. doi: $10.1039 / \mathrm{c} 5 \mathrm{ra} 14889 \mathrm{~h}$

(16) Shi, Y. Q.; Zhou, K. Q.; Wang, B. B.; Jiang, S. H.; Qian, X. D.; Gui, Z.; Yuen, R. K. K.; Hu, Y. J. Mater. Chem. A 2014, 2 (2), 535. doi: 10.1039/c3ta13409a

(17) Singh, S.; Khare, N. Mater. Lett. 2015, 161, 64. doi: 10.1016/j. matlet.2015.07.035

(18) Ao, Y. H.; Wang, K. D.; Wang, P. F.; Wang, C.; Hou, J. Appl. Catal. B 2016, 194, 157. doi: 10.1016/j.apcatb.2016.04.050

(19) Ao, Y. H.; Bao, J. Q.; Wang, P. F.; Wang, C.; Hou, J. J. Colloid Interface Sci. 2016, 476, 71. doi: 10.1016/j.jcis.2016.05.021

(20) Zhao, W.; Liu, Y.; Wei, Z. B.; Yang, S. G.; He, H.; Sun, C. Appl. Catal. B 2016, 185, 242. doi: 10.1016/j.apcatb.2015.12.023

(21) Peng, T. Y.; Ke, D. N.; Zeng, P.; Zhang, X. H.; Fan, K. Acta Phys. -Chim. Sin. 2011, 27 (9), 2160. [彭天右, 柯丁宁, 曾 鹏, 张晓虎, 范 科. 物理化学学报, 2011, 27 (9), 2160.] doi: 10.3866/PKU.WHXB20110913

(22) Min, S. X.; Lü, G. X. Acta Phys. -Chim. Sin. 2011, 27 (9), 2178. [敏世雄, 吕功煊. 物理化学学报, 2011, 27 (9), 2178.] doi: 10.3866/PKU.WHXB20110904

(23) Guo, X. Y.; Chen, C. F.; Song, W. Y.; Wang, X.; Di, W. H.; Qin, W. P. J. Mol. Catal. A: Chem. 2014, 387, 1. doi: 10.1016/j. molcata.2014.02.020

(24) Sathishkumar, P.; Mangalaraja, R.V.; Anandan, S.; Ashokkumar, M. Chem. Eng. J. 2013, 220, 302. doi: 10.1016/j.cej.2013.01.036

(25) Li, W. J.; Li, D. Z.; Zhang, W. J.; Hu, Y.; He, Y. H.; Fu, X. Z. J. Phys. Chem. C 2010, 14 (5), 2154. doi: 10.1021/jp9066247

(26) Jia, Y. S.; Yang, J. X.; Zhao, D.; Han, H. X.; Li, C. J. Energy Chem. 2014, 23 (4), 420. doi: 10.1016/S2095-4956(14)60167-4

(27) Panda, R.; Rathore, V.; Rathore, M.; Shelke, V.; Badera, N.; Chandra, L. S. S.; Jain, D.; Gangrade, M.; Shripati, T.; Ganesan, V. Appl. Surf. Sci. 2012, 258 (12), 5086. doi: 10.1016/j. 
apsusc.2012.01.131

(28) Dong, X.; Tao, J.; Li, Y. Y.; Wang, T.; Zhu, H. Acta Phys. -Chim. Sin. 2009, 25 (9), 1874. [董 祥, 陶 杰, 李莹茎, 汪 涛, 朱 宏. 物理化学学报, 2009, 25 (9), 1874.] doi: 10.3866/PKU. WHXB20090913

(29) Qi, Q.; Wang, Y. Q.; Wang, S. S.; Qi, H. N.; Wei, T.; Sun, Y. M. Acta Phys. -Chim. Sin. 2015, 31 (12), 2332. [齐 齐, 王育乔, 王 莎莎, 祁吴楠, 卫 涛, 孙岳明. 物理化学学报, 2015, 31 (12), 2332.] doi: 10.3866/PKU.WHXB201510202

(30) Zhang, J.; Nosaka, Y. Appl. Catal. B 2015, 166, 32. doi: 10.1016/ j.apcatb.2014.11.006

(31) Chai, B.; Zhou, H.; Zhang, F.; Liao, X.; Ren, M. X. Mater. Sci. Semicond. Process. 2014, 23, 151. doi: 10.1016/j. mssp.2014.02.021
(32) Lam, S. M.; Sin, J. C.; Abdullah, A. Z.; Mohamed, A. R. Sep. Purif. Technol. 2014, 132, 378. doi: 10.1016/j. seppur.2014.05.043

(33) Jia, H. M.; He, W. W.; Wamer, W. G.; Han, X. N.; Zhang, B. B.; Zhang, S.; Zheng, Z.; Xiang, Y.; Yin, J. J. J. Phys. Chem. C 2014, 118, 21447. doi: 10.1021/jp505783y

(34) Li, R. G.; Wang, X. L.; Jin, S. Q.; Zhou, X.; Feng, Z. C.; Li, Z.; Shi, J. Y.; Zhang, Q.; Li, C. Sci. Rep. 2015, 5, 13475. doi: 10.1038/srep13475

(35) Zheng, X. J.; Wei, Y. J.; Wei, L. F.; Xie, B.; Wei, M. B. Int. J. Hydrog. Energy 2010, 35 (21), 11709. doi: 10.1016/j. ijhydene.2010.08.090

(36) Catano, F. A.; Valencia, S. H.; Hincapie, E. A.; Restrepo, G. M.; Marin, J. M. Lat. Am. Appl. Res. 2012, 42 (1), 33. 